\title{
SOME VARIETIES CONTAINING RELATION ALGEBRAS
}

BY

\author{
ROGER MADDUX
}

\begin{abstract}
Three varieties of algebras are introduced which extend the variety $R A$ of relation algebras. They are obtained from $R A$ by weakening the associative law for relative product, and are consequently called nonassociative, weakly-associative and semiassociative relation algebras, or $N A, W A$, and $S A$, respectively. Each of these varieties arises naturally in solving various problems concerning relation algebras. We show, for example, that $W A$ is the only one of these varieties which is closed under the formation of complex algebras of atom structures of algebras, and that $W A$ is the closure of the variety of representable $R A$ 's under relativization. The paper also contains a study of the elementary theories of these varieties, various representation theorems, and numerous examples.
\end{abstract}

0. Introduction. Relation algebras ( $R A$ 's) have a binary operation ; which serves as an abstract algebraic analogue of the relative product of binary relations. (The relative product of $R, S \subseteq U \times U$, is $R \mid S=\{\langle x, z\rangle:\langle x, y\rangle \in R$ and $\langle y, z\rangle \in S$ for some $y \in U\}$.) The relative product is associative, and one of the postulates for $R A$ 's is that ; is associative. The associativity of relative product can be expressed by a sentence in a first-order language with binary relation symbols, namely

$$
\forall_{x} \forall_{y}\left[\exists_{z}\left(\exists_{y}(R x y \wedge S y z) \wedge T z y\right) \leftrightarrow \exists_{z}\left(R x z \wedge \exists_{x}(S z x \wedge T x y)\right)\right] .
$$

Although this sentence has three variables, it cannot be proved from the ordinary axioms of first-order logic without using four variables. In contrast, all the other postulates for relation algebras can not only be expressed but proved using only three variables. (These facts were first proved by Tarski. For a proof that (1) requires four variables to prove, see [3].) Tarski asked whether there are any other equations whose translations into first-order sentences can be expressed and proved using only three variables, but which are not derivable from the postulates for $R A$ 's without using the associativity of ;. There are such equations. One of them is a special case of the associative law for ; called the "semiassociative law",

$$
x ;(1 ; 1)=(x ; 1) ; 1 .
$$

The class $S A$ of semiassociative relation algebras is defined by the postulates for $R A$ 's with the associative law for ; replaced by (2). This class properly includes $R A$, as will be shown in this paper.

Received by the editors November 12, 1980.

1980 Mathematics Subject Classification. Primary 03G25, 06E99.

Key words and phrases. Relation algebra, Boolean algebra with operators, relativization, complex algebra. 
About $90 \%$ of the theorems for $R A$ 's proved in Part I of [1] for $R A$ 's carry over to $S A$ 's. In fact, about $80 \%$ of those theorems do not even require the semiassociative law, and hence apply to the even wider class of nonassociative relation algebras $N A$. The definition of $N A$ is obtained from that of $R A$ by omitting the associative law. $N A$ 's are Boolean algebras with operators in the sense of [6, Definition 2.13]. Consequently the Representation Theorem 3.10 of [6] can be applied. One of the goals of this paper is to obtain a more explicit form of this theorem for $N A, S A$, and $R A$. To do so we introduce the appropriate notions of complex algebra and atom structure, and study some of their basic properties. In the course of this study another class of algebras naturally arises, namely $W A$, the weakly-associative relation algebras. The definition of $W A$ is obtained from that of $R A$ by replacing the associative law for ; by

$$
(1 ' x) ;(1 ; 1)=((1 ' x) ; 1) ; 1 .
$$

Note that (3) is a special case of (2). We shall see that $R A \subset S A \subset W A \subset N A$. Another goal of this paper is to introduce the notion of relativization for $N A$ 's and prove a new result for $R A$ 's involving relativization. It turns out, however, that $W A$ is exactly the class of algebras for which this theorem holds.

The paper is organized as follows. In $\$ 1$ we define the algebras which concern us and prove a few elementary properties of $N A$ 's. The duality theorems for $R A$ 's in [1] carry over to $N A$ 's and are given in $\$ 1$. In $\$ 2$ we define complex algebras of certain relational structures and characterize those structures which give use to $N A$ 's, $W A$ 's, $S A$ 's, and $R A$ 's. Some examples appear at the end of the section. Atom structures are defined and studied in $\S 3$. Theorems 3.8-3.10 in that section are reasons for introducing $W A$. Examples of $N A$ 's which are not $W A$ 's also appear there. Results from $\S \S 2$ and 3 are combined with some theorems of [6] in $\S 4$ to yield an explicit form of the Representation Theorem (4.3). $\$ 5$ deals with relativization. The major result there is that $W A$ is the class of subalgebras of relativized representable $R A$ 's (5.20).

In set-theoretical, logical, and general algebraic notation and terminology we follow [4]. For the theory of Boolean algebras our reference is [16]. The most important reference for this paper is [6]. We shall use several of the theorems in that paper, and refer the reader there for the definitions of Boolean algebra with operators, and of conjugate, self-conjugate, normal, monotone, additive, and completely additive operators on a Boolean algebra.

\section{Definitions and elementary properties.}

DEFINITION 1.1. $\mathfrak{A}$ is a relation-type algebra iff $\mathfrak{A}=\left\langle A,+, \cdot,-, 0,1, ;, \cup, 1^{\prime}\right\rangle$, where,$+ \cdot$, and ; are binary operations on $A,-$ and $\cup$ are unary operations on $A$, and $0,1,1$ ' $\in A$. The reduct $\mathfrak{B} \mathfrak{I} \mathfrak{A}=\langle A,+, \cdot,-, 0,1\rangle$ is called the Boolean part of $\mathfrak{A}$. The Boolean operations of $\mathfrak{A}$ are,$+ \cdot$, and - . For all $x, y \in A$ let $x+y=$ $-((-x) ;(-y))$, and $0^{\prime}=-1^{\prime} . x+y$ is the relative sum of $x$ and $y, x ; y$ is the relative product of $x$ and $y$, and $x \cup$ is the converse of $x$. The Peircean (or relative) operations of $\mathfrak{A}$ are,$+ ;$, and $~ '$. The elements $0,1,0$, and $1^{\prime}$ are called the zero element, unit element, diversity element, and identity element, respectively. For each $x \in A$ let $l_{x}$, 
$r_{x}, l_{x}^{\cup}$, and $r_{x}^{\cup}$ be the functions mapping $A$ into $A$ which are defined as follows for all $y \in A$ :

$$
\begin{array}{ll}
l_{x}(y)=x ; y, & r_{x}(y)=y ; x, \\
l_{x}^{\cup}(y)=x^{\cup} ; y, & r_{x}^{\cup}(y)=y ; x^{\cup} .
\end{array}
$$

Parentheses will be omitted from expressions involving the operations of a relation-type algebra according to the convention that the operations should be performed in the following order: $\cup,-, ;, \cdot,+,+$. Thus, for example, $-x^{\cup}=$ $-\left(x^{\cup}\right),-x ;-y=(-x) ;(-y)$, and $v+w ; x \cdot y+z=(v+((w ; x) \cdot y))+z$. When the same binary operation occurs several times, the calculation proceeds from left to right, e.g. $x ; y ; z=(x ; y) ; z$.

Let $\mathfrak{U}$ be a relation-type algebra. If $\mathfrak{B} \mathfrak{l} \mathfrak{A}$ is a Boolean algebra, then we will apply terminology from the theory of Boolean algebras to $\mathfrak{A}$, with the understanding that we are referring to $\mathfrak{B} \mathfrak{l} \mathfrak{A}$. For example, $\mathfrak{A}$ is complete iff $\mathfrak{B l} \mathfrak{A}$ is complete, and $x \in A$ is an atom of $\mathfrak{A}$ iff $x$ is an atom of $\mathfrak{B} \mathfrak{l} \mathfrak{A}$.

DEFINITION 1.2. A nonassociative relation algebra is a relation-type algebra $\mathfrak{A}=\langle A$, $\left.+, \cdot,-, 0,1, ;, \cup, 1^{\prime}\right\rangle$ which satisfies the following postulates:

(1) $\mathfrak{B l} \mathfrak{A}$ is a Boolean algebra,

(2) $x=x ; 1^{\prime}=1$ '; $x$ for every $x \in A$,

(3) $l_{x}$ and $l_{x}^{\cup}$ are conjugate for every $x \in A$, i.e. $x ; y \cdot z=0$ iff $x^{\cup} ; z \cdot y=0$ for all $x, y, z \in A$,

(4) $r_{x}$ and $r_{x}^{\cup}$ are conjugate for every $x \in A$, i.e. $y ; x \cdot z=0$ iff $z ; x^{\cup} \cdot y=0$ for all $x, y, z \in A$.

$N A$ is the class of all nonassociative relation algebras.

$\mathfrak{A}$ is a weakly-associative relation algebra iff $\mathfrak{U} \in N A$ and

$$
(1 ' x) ; 1 ; 1=(1 ' x) ; 1 \text { for every } x \in A \text {. }
$$

$W A$ is the class of all weakly-associative relation algebras.

$\mathfrak{A}$ is a semiassociative relation algebra iff $\mathfrak{A} \in N A$ and

$$
x ; 1 ; 1=x ; 1 \text { for every } x \in A .
$$

$S A$ is the class of all semiassociative relation algebras.

$\mathfrak{U}$ is a relation algebra iff $\mathfrak{U} \in N A$ and

$$
x ; y ; z=(x ; y) ; z \text { for all } x, y, z \in A .
$$

$R A$ is the class of all relation algebras.

Corollary 1.3. (1) $S A \subseteq W A \subseteq N A$.

(2) $R A \subseteq N A$.

We will use " $B A$ ", " $N A$ ", " $W A$ ", " $S A$ ", and " $R A$ " as abbreviations of the phrases "Boolean algebra", "nonassociative relation algebra", "weakly-associative relation algebra", "semiassociative relation algebra," and "relation algebra", respectively. Postulates 1.2(2)-(7) will be referred to respectively as the identity law (IL), left Peircean law (LPL), right Peircean law (RPL), weak associative law (WL), semiassociative law (SL), and associative law (AL). 
It will be shown below that $1=1 ; 1$ in every $N A$. Hence, relative to postulates 1.2(1)-(4), the WL and SL are special cases of the $\mathrm{AL}$, namely $\left(1^{\prime} \cdot x\right) ; 1 ; 1=$ $(1 ' x) ;(1 ; 1)$ and $x ; 1 ; 1=x ;(1 ; 1)$, respectively. This is the reason for the use of the terms "weakly-associative" and "semiassociative" in 1.2.

Postulates 2.1(1)-(4), (7) were first used as a definition of $R A$ in [7]. The definition of $R A$ in [1] is proved in Theorem 2.2 of [1] to be equivalent to the one adopted here.

THEOREM 1.4. Let $\mathfrak{A}$ be a relation-type algebra with $\mathfrak{B} \mathfrak{I} \mathfrak{A} \in B A$.

(1) The following conditions are equivalent:

(a) $\mathfrak{A}$ satisfies the $L P L$,

(b) $x ;\left(y \cdot-\left(x^{\cup} ; z\right)\right) \leqslant x ; y \cdot-z$ and $x^{\cup} ;(y \cdot-(x ; z)) \leqslant x^{\cup} ; y \cdot-z$ for all $x, y$, $z \in A$,

(c) $x ; 0=0, x ; y \cdot z \leqslant x ;\left(y \cdot x^{\cup} ; z\right)$ and $x^{\cup} ; y \cdot z \leqslant x^{\cup} ;(y \cdot x ; z)$ for all $x, y$, $z \in A$.

(2) The following conditions are equivalent:

(a) $\mathfrak{A}$ satisfies the RPL,

(b) $\left(y \cdot-\left(z ; x^{\cup}\right)\right) ; x \leqslant y ; x \cdot-z$ and $(y \cdot-(z ; x)) ; x^{\cup} \leqslant y ; x^{\cup} \cdot-z$ for all $x, y$, $z \in A$,

(c) $0 ; x=0, y ; x \cdot z \leqslant\left(y \cdot z ; x^{\cup}\right) ; x$, and $y ; x^{\cup} \cdot z \leqslant(y \cdot z ; x) ; x^{\cup}$ for all $x, y$, $z \in A$.

Proof. Use Theorem 1.15 of [6]. Notice that if $l_{x}$ (or $r_{x}$ ) is normal for every $x \in A$, then so is $l_{x}^{\cup}$ (or $\left.r_{x}^{\cup}\right)$.

COROllary 1.5. NA, WA, SA, and $R A$ are finitely based equational classes; $H S P N A=N A, H S P W A=W A, H S P S A=S A$, and HSPRA $=R A$.

Proof. Use 1.4 and the fact that $B A$ 's can be characterized by equations when + , $\cdot,-, 0$, and 1 are taken as fundamental.

We now turn to examples of $R A$ 's which motivated the abstract definition of $R A$ and gave rise to the concept of representability. Let $U$ be a set. Notice that $\operatorname{Sb}(U \times U)$, the set of subsets of $U \times U$, is closed under $U, \cap, U \times U \sim$ (complementation with respect to $U \times U)$, | (defined by $R \mid S=\{\langle u, w\rangle:\langle u, v\rangle \in R$ and $\langle v, w\rangle \in S$, for some $v \in U\}$ for all $R, S \subseteq U \times U$ ), and ${ }^{-1}$ (where $R^{-1}=$ $\{\langle v, u\rangle:\langle u, v\rangle \in R\})$. Furthermore, if $E$ is an arbitrary binary relation, then $\mathrm{Sb} E$ is closed under $\cup, \cap,{ }_{E} \sim, \mid$, and ${ }^{-1}$ iff $E$ is an equivalence relation.

DEFINITION 1.6. Let $U$ be any set. Then $\operatorname{Re} U$ is the set of all (binary) relations on $U$, i.e., $\operatorname{Re} U=\operatorname{Sb}(U \times U)$, and

$$
\Re \text { e } U=\left\langle\operatorname{Re} U, \cup, \cap, U \times U \sim, \varnothing, U \times U, \mid,{ }^{-1}, \mathrm{Id}_{U}\right\rangle
$$

is the algebra of relations on $U$.

Let $E$ be any equivalence relation. Then

$$
\varsigma_{\mathfrak{b}} E=\left\langle\mathrm{Sb} E, \cup, \cap,,_{E} \sim, \varnothing, E, \mid,{ }^{-1}, \operatorname{Id}_{\mathrm{Fd} E}\right\rangle
$$

is the algebra of subrelations of $E$. 
THEOREM 1.7. Let $U$ be $a$ set and $E$ an equivalence relation.

(1) $\Re$ e $U \in R A$ and $\subseteq \mathfrak{b} E \in R A$.

(2) $\Re$ e $U$ is a one-element algebra iff $U=\varnothing$, and $\subseteq \mathfrak{b} E$ is a one-element algebra iff $E=\varnothing$.

(3) For every set $V$, e $V \cong \Re$ e $U$ iff $|V|=|U|$.

(4) $\Re \mathfrak{e} U=\subseteq \mathfrak{b} E$ iff $U \times U=E$.

(5) $\subseteq \mathfrak{b} E \cong P_{U \in(\mathrm{Fd} E) / E} \Re \in$

Proof. Parts (1)-(4) are easy to check. For part (5), define a function $f$ mapping $\mathrm{Sb} E$ into the direct product of the equivalence classes of $E$ as follows: if $R \in \mathrm{Sb} E$ then $f(R)=\langle R \cap(U \times U): U \in(\mathrm{Fd} E) / E\rangle$. It is easy to check that $f$ is an isomorphism.

DEFINITION 1.8. A $R A \mathfrak{A}$ is representable if it is isomorphic to a subalgebra of $\subseteq \mathfrak{b} E$, for some equivalence relation $E$. $R R A$ is the class of all representable $R A$ 's, i.e. $R R A=I S\{\subseteq \mathfrak{b} E: E$ is an equivalence relation $\}$.

THEOREM 1.9. (1) $R R A \subseteq R A$.

(2) $\Re$ e $U \in R R A$ for every set $U$.

(3) $\subseteq \mathfrak{b} E \in R R A$ for every equivalence relation $E$.

(4) $H S P R R A=R R A$.

Proof. Parts (1)-(3) and $S P R R A=R R A$ are easy to prove using 1.7. For a proof of $H R R A=R R A$, see Theorem 2.5(i) of [17], or Corollary 11 of [11].

The rest of this section will be devoted to some elementary properties of $N A$ 's which will be needed in this paper.

THEOREM 1.10. Let $\mathfrak{A}$ be a relation-type algebra in which the Peircean laws hold. Then ; is normal and completely additive.

Proof. The operation ; is normal and completely additive iff $l_{x}$ and $r_{x}$ are normal and completely additive for all $x \in A$, so the theorem follows immediately from Theorem 1.14 of [6].

THEOREM 1.11. Let $\mathfrak{A} \in N A$. Then $U$ is self-conjugate, normal, and completely additive.

Proof. In view of Theorem 1.14 of [6], it suffices to show $U$ is self-conjugate, i.e. $x^{\cup} \cdot y=0$ iff $x \cdot y^{\cup}=0$ for all $x, y \in A$. Each of the following formulas is equivalent to the one preceding it (if any) for the reason given to the right.

$$
\begin{aligned}
& 0=x^{\cup} \cdot y \\
& 0=x^{\cup} ; 1^{\prime} \cdot y \quad \mathrm{IL} \\
& 0=x ; y \cdot 1 \text {, LPL } \\
& 0=1^{\prime} ; y^{\cup} \cdot x \quad \text { RPL } \\
& 0=x \cdot y^{U} \quad \text { IL. }
\end{aligned}
$$

THEOREM 1.12. Every NA is a normal BA with operators.

Proof. Use 1.10 and 1.11 . 
TheOREM 1.13. Let $\mathfrak{A} \in N A$. Then for all $w, x, y, z \in A$ we have

(1) $(x+y) ; z=x ; z+y ; z$,

(2) $x ;(y+z)=x ; y+x ; z$,

(3) if $w \leqslant x$ and $y \leqslant z$, then $w ; y \leqslant x ; z$,

(4) $x \leqslant x ; 1$ and $x \leqslant 1 ; x$,

(5) $1=1 ; 1$,

(6) $(x+y)^{\cup}=x^{\cup}+y^{\cup}$,

(7) $0^{\cup}=0$,

(8) $x^{\cup \cup}=x$,

(9) $1^{\cup}=1$,

(10) $(-x)^{\cup}=-x^{\cup}$,

(11) $(x \cdot y)^{\cup}=x^{\cup} \cdot y^{\cup}$,

(12) $x \leqslant y$ iff $x^{\cup} \leqslant y^{\cup}$,

(13) $(x ; y)^{\cup}=y^{\cup} ; x^{\cup}$,

(14) $1^{\prime} \cup=1$ and $0^{\prime} \cup=0$,

(15) if $x, y \leqslant 1$ ' then $x ; y=x \cdot y$,

(16) if $x \leqslant 1$ 'then $x^{\cup}=x$.

Proof. (1)-(3) follow immediately from 1.10. Using (3) and the identity law, we get $x=x ; 1^{\prime} \leqslant x ; 1$ and $x=1 ; x \leqslant 1 ; x$, so (4) holds. Since $1 ; 1 \leqslant 1$, we get (5) from (4). (6) and (7) follow from 1.11. For (8) we first notice that the following formulas are equivalent:

$$
\begin{aligned}
& 0=x \cdot y \\
& 0=x ; 1^{\prime} \cdot y \quad \text { IL } \\
& 0=x^{\cup} ; y \cdot 1 \text {, LPL } \\
& 0=x^{\cup \cup} ; 1^{\prime} \cdot y \quad \text { LPL } \\
& 0=x^{\cup \cup} \cdot y \text {. }
\end{aligned}
$$

We get $x^{\cup \cup}=x$ by first replacing $y$ by $-x$, and then by $-x \cup \cup$. Note that $1=1+1^{\cup}$, so by (6) and (8) we get (9) as follows: $1=1+1^{\cup}=1^{\cup U}+1^{\cup}=$ $\left(1^{\cup}+1\right)^{\cup}=1^{\cup}$. For (10) we first get $-x \cdot x^{\cup \cup}=0$ from $-x \cdot x=0$ by (8), and then $(-x)^{\cup} \cdot x^{\cup}=0$ by 1.11. Also, by (6) and (9), $(-x)^{\cup}+x^{\cup}=(-x+x)^{\cup}=1^{\cup}=1$. It follows that $(-x)^{\cup}=-x^{\cup}$. (11) follows from (6) and (10), and (12) follows from (6) and (8). For (13), we first note that the following formulas are equivalent:

$$
\begin{array}{ll}
0=(x ; y)^{\cup} \cdot z & \\
0=x ; y \cdot z^{\cup} & 1.11 \\
0=x^{\cup} ; z^{\cup} \cdot y & \text { LPL } \\
0=y ; z \cdot x^{\cup} & \text { RPL } \\
0=y^{\cup} ; x^{\cup} \cdot z & \text { LPL. }
\end{array}
$$

Let $z=-(x ; y)^{\cup}$, and then let $z=-\left(y^{\cup} ; x^{\cup}\right)$. It follows that $(x ; y)^{\cup}=y^{\cup} ; x^{\cup}$. By (13), (8), and the IL, we have $1^{\prime \cup}=1^{, \cup} ; 1^{\prime \cup \cup}=\left(1^{\prime}, 1^{\prime}\right)^{\cup}=1, \cup \cup=1$, and hence, by 
(10), $0^{\prime} \cup=\left(-1^{\prime}\right)^{\cup}=-1^{\prime} \cup=-1^{\prime}=0$ '. Thus (14) holds. To prove (15) and (16), we assume $x, y \leqslant 1$ ' and proceed as follows:

$$
\begin{aligned}
x ; y & \leqslant x ; 1^{\prime} \cdot 1^{\prime} ; y & & \text { (3) } \\
& =x \cdot y & & \text { IL } \\
& =x ; 1^{\prime} \cdot y & & \text { IL } \\
& \leqslant x ;\left(1^{\prime} \cdot x^{\cup} ; y\right) & & 1.4(1) \\
& \leqslant x ;\left(1^{\cup} ; y\right) & & (3),(12) \\
& =x ; y & & \text { (14), IL }
\end{aligned}
$$

so (15) holds. We have

$$
\begin{aligned}
x & =1^{\prime} ; x \cdot 1^{\prime} & & \text { IL } \\
& \leqslant\left(1^{\prime} \cdot 1^{\prime} ; x^{\cup}\right) ; x & & 1.4(2) \\
& \leqslant 1^{\prime} ; x^{\cup} ; 1^{\prime} & & \text { (3) } \\
& =x^{\cup} & & \text { IL }
\end{aligned}
$$

so, by (12) and (8), we also get $x^{\cup} \leqslant x^{\cup \cup}=x$. Thus (16) holds, and the proof is complete.

Corollary 1.14. $R A \subseteq S A$.

Proof. Use 1.13(5).

DEFINITION 1.15. For every relation-type algebra

$$
\mathfrak{U}=\left\langle A,+, \cdot,-, 0,1, ;,^{\cup}, 1^{\prime}\right\rangle
$$

let

$$
\mathfrak{A}^{\cup}=\left\langle A,+, \cdot,-, 0,1, ;^{\cup},{ }^{\cup}, 1^{\prime}\right\rangle
$$

where $x ;{ }^{\cup} y=y ; x$ for all $x, y \in A$,

$$
\mathfrak{A}^{-}=\left\langle A, \cdot,+,-, 1,0,+,,^{\cup}, 0^{\prime}\right\rangle,
$$

and

$$
\mathfrak{U}^{\prime}=\left\langle A, \cdot,+,-, 1,0,+\stackrel{\cup}{ },^{\cup}, 0^{\prime}\right\rangle,
$$

where $x+\cup^{\prime} y=y+x$ for all $x, y \in A$, and let $x^{\prime}=-x^{\cup}$ for every $x \in A$.

THEOREM 1.16. Let $\mathfrak{A} \in N A$. Then $\mathfrak{A} \cong \mathfrak{A}^{\cup} \cong \mathfrak{U}^{-} \cong \mathfrak{A}^{\prime}=\left(\mathfrak{U}^{-}\right)^{\cup}=\left(\mathfrak{A}^{\cup}\right)^{-}$. In particular, the operations $\cup,-$, and 'are involutions (i.e. $x=x^{\cup \cup}=--x=x^{\prime \prime}$ ) and are isomorphisms between these algebras as indicated in the following diagram:

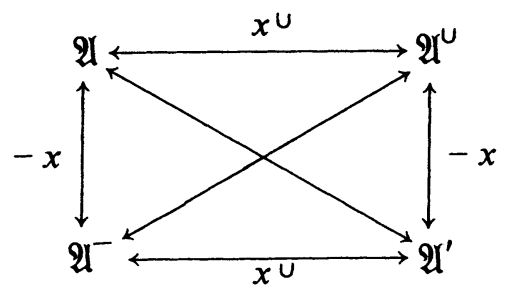


Proof. We get $x=x \cup \cup=-x=x^{\prime \prime}$ from 1.13(8), (10), so $\cup,-$, and ' are one-to-one and onto. The fact that they are also homomorphisms can be expressed by a number of identities, each of which can be easily derived from 1.13(6)-(11), (13), (14).

Given any theorem for $N A$ 's, we can obtain three other (not necessarily different) theorems for $N A$ 's by changing the operations in the original theorem to the corresponding operations of any one of the dual algebras $\mathfrak{A}^{\cup}, \mathfrak{A}^{-}$, and $\mathfrak{X}^{\prime}$. (Thus there are three duality principles, which are discussed more fully in [1].) For example, if $\mathfrak{A} \in N A$, then $\mathfrak{U} \in W A$ iff $\mathfrak{A}$ satisfies any (or all) of the following identities: $\left(1^{\prime} \cdot x\right) ; 1 ; 1=\left(1^{\prime} \cdot x\right) ; 1,1 ;\left(1 ;\left(1^{\prime} \cdot x\right)\right)=1 ;\left(1^{\prime} \cdot x\right),\left(0^{\prime}+x\right)+0+0=$ $\left(0^{\prime}+x\right)+0,0+\left(0+\left(0^{\prime}+x\right)\right)=0+\left(0^{\prime}+x\right)$.

\section{Complex algebras.}

Definition 2.1. Let $\mathfrak{U}=\langle U, C, f, I\rangle$ where $C \subseteq{ }^{3} U, f \in{ }^{U} U$, and $I \subseteq U$. Then (5) $\mathfrak{m} \mathfrak{u}=\left\langle\mathrm{Sb} U, \cup, \cap,{ }_{U} \sim, \varnothing, U, ;, \cup, I\right\rangle$ where $X ; Y=\{z:\langle x, y, z\rangle \in C$ for some $x \in X, y \in Y\}$ and $X^{\cup}=\{f x: x \in X\}$ for all $X, Y \in \operatorname{Sb} U$. $\mathfrak{c} \mathfrak{m} \mathfrak{U}$ is called the complex algebra of $\mathfrak{u}$.

The complex algebra of a structure is defined in Definition 3.8 of [6] for the case in which the structure has arbitrarily many finitary relations. If we were to treat $f$ as a binary relation, then 2.1 would be a special case of the definition in [6].

The notion of complex algebra also occurs in universal algebra (see [2, p. 379]), but with a different meaning: the Boolean operations are not included, and the notion is defined only for algebras (structures with functions and no relations).

THEOREM 2.2. Let $\mathfrak{U}=\langle U, C, f, I\rangle$ where $C \subseteq{ }^{3} U, f \in{ }^{U} U$, and $I \subseteq U$.

(1) $\mathfrak{s} \mathfrak{m} \mathfrak{U}$ is a relation-type algebra, $\mathfrak{B l} \mathfrak{C} \mathfrak{m} \mathfrak{U}$ is a complete atomic $B A$, and the operations; and $\cup$ are normal and completely additive.

(2) $\mathfrak{S} \mathfrak{m} \mathfrak{U} \in N A$ iff $\mathfrak{U}$ satisfies the following conditions for all $x, y, z \in U$.

(a) If $\langle x, y, z\rangle \in C$ then $\langle f x, z, y\rangle \in C$,

(b) if $\langle x, y, z\rangle \in C$ then $\langle z, f y, x\rangle \in C$,

(c) $x=y$ iff there is some $w \in I$ such that $\langle x, w, y\rangle \in C$.

(3) $\mathfrak{S} \mathfrak{m} \mathfrak{U} \in W A$ iff $\mathfrak{U}$ satisfies (a)-(c) and

(d) if $w \in I$ and $\langle w, x, x\rangle,\langle x, y, z\rangle \in C$, then $\langle w, z, z\rangle \in C$.

(4) $\mathfrak{E} \mathfrak{m} \mathfrak{U} \in S A$ iff $\mathfrak{U}$ satisfies (a)-(c) and

(e) if $\langle v, w, x\rangle,\langle x, y, z\rangle \in C$ then there is some $u \in U$ such that $\langle v, u, z\rangle \in C$.

(5) $\mathfrak{E} \mathfrak{m} \mathfrak{U} \in R A$ iff $\mathfrak{U}$ satisfies (a)-(c) and

(f) if $\langle v, w, x\rangle,\langle x, y, z\rangle \in C$ then there is some $u \in U$ such that $\langle v, u, z\rangle$, and $\langle w, y, u\rangle \in C$.

Proof. (1) is easy to check. (See Theorem 3.3 of [6].)

(2) Assume $\mathfrak{E} \mathfrak{m} \mathfrak{u} \in N A$. We first prove (a) and (b). Let $\langle x, y, z\rangle \in C$. Then $\{z\} \subseteq\{x\} ;\{y\}$, or, equivalently, $\{x\} ;\{y\} \cap\{z\} \neq \varnothing$. Applying the LPL yields $\{f x\} ;\{z\} \cap\{y\}=\{x\}^{\cup} ;\{z\} \cap\{y\} \neq \varnothing$, so $\langle f x, z, y\rangle \in C$. Similarly, the RPL yields $\langle z, f y, x\rangle \in C$.

If $w \in I$ and $\langle x, w, y\rangle \in C$, then $y \in\{x\} ;\{w\} \subseteq\{x\} ; I=\{x\}$ by the IL, so $x=y$. Conversely, if $x=y$ then $y \in\{x\}=\{x\} ; I$, so there is some $w \in I$ such that $\langle x, w, y\rangle \in C$. Thus (c) holds. 
To proceed further we need the following.

LEMMA. If $\mathfrak{U}$ satisfies (a) and (c), then ffx $=x$ for every $x \in U$.

Proof. Let $x \in U$. Then $\langle x, w, x\rangle \in C$ for some $w \in I$ by (c). Using (a) twice we get $\langle f x, x, w\rangle \in C$ and then $\langle f f x, w, x\rangle \in C$. Consequently $f f x=x$ by (c).

Now we assume $\mathfrak{U}$ satisfies (a)-(c) and show $\mathbb{E} \mathfrak{m} \mathfrak{U} \in N A$. Since $\mathfrak{B} \mathfrak{I} \mathfrak{c} \mathfrak{m} \mathfrak{U} \in B A$ we need only prove the identity and Peircean laws. Let $X \subseteq U$. If $x \in X$, then $\langle x, w$, $x\rangle \in C$ for some $w \in I$ by (c), so $x \in\{x\} ;\{w\} \subseteq X$; I. Thus $X \subseteq X ; I$. On the other hand, if $y \in X$; $I$, then $\langle x, w, y\rangle \in C$ for some $x \in X$ and $w \in I$, but $x=y$ by (c), so $y \in X$. Thus $X ; I \subseteq X$, and we have $X=X$; I. Let $x \in X$. Then $\langle f x, w, f x\rangle \in C$ for some $w \in I$ by (c). We get $\langle f f x, f x, w\rangle \in C$ by (a), and then $\langle w, x, x\rangle=\langle w, f f x$, $f f x\rangle \in C$ by the lemma and (b). Thus $x \in\{w\} ;\{x\} \subseteq I ; X$, so we have shown $X \subseteq I ; X$. Now let $y \in I ; X$, i.e. $\langle w, x, y\rangle \in C$ for some $w \in I$ and $x \in X$. Then $\langle y$, $f x, w\rangle \in C$ by (b), so $\langle f y, w, f x\rangle \in C$ by (a). Applying (c) we get $f y=f x$, so $y=f f y=f f x=x$ by the lemma. Thus $y \in X$, which shows $I ; X \subseteq X$. The proof of the identity law is complete.

The Peircean laws hold in $\sqrt{ } \mathrm{m} \mathfrak{U}$ iff the following formulas are equivalent for all $X, Y, Z \in \mathrm{Sb} U: X ; Y \cap Z \neq \varnothing, X^{\cup} ; Z \cap Y \neq \varnothing, Z ; Y^{\cup} \cap X \neq \varnothing$. We shall only prove the first two are equivalent; the other equivalences can be proved similarly.

Let $X ; Y \cap Z \neq \varnothing$. Then $\langle x, y, z\rangle \in C$ for some $x \in X, y \in Y, z \in Z$. By (a), $\langle f x, z, y\rangle \in C$, so $\varnothing \neq\{f x\} ;\{z\} \cap\{y\}=\{x\}^{\cup} ;\{z\} \cap\{y\} \subseteq X^{\cup} ; Z \cap Y$. Now suppose $X^{\cup} ; Z \cap Y \neq \varnothing$. Then there are $x \in X, y \in Y, z \in Z$ such that $\langle f x, z$, $y\rangle \in C$. By the lemma and (a), $\langle x, y, z\rangle=\langle f f x, y, z\rangle \in C$, so $X ; Y \cap Z \neq \varnothing$.

(3) Assume $\mathbb{E} \mathfrak{m} \mathfrak{U} \in W A$. Then (a)-(c) hold by (2). Suppose $w \in I$ and $\langle w, x, x\rangle$, $\langle x, y, z\rangle \in C$. Then $\{x\} \subseteq\{w\} ;\{x\}$ and $z \in\{x\} ;\{y\}$, so $z \in\{w\} ;\{x\} ;\{y\} \subseteq$ $\{w\} ; U ; U=\{w\} ; U$ by the WL. Consequently $\langle w, v, z\rangle \in C$ for some $v \in U$. But $v=z$ since $z \in\{w\} ;\{v\} \subseteq I ;\{v\}=\{v\}$ by the IL, so $\langle w, z, z\rangle \in C$. Thus (d) holds.

Assume $\mathfrak{U}$ satisfies (a)-(d). Then $\mathbb{E}_{\mathfrak{m}} \mathfrak{U} \in N A$ by (2), so we need only prove the WL for $\mathbb{E} \mathfrak{m}$ U. Let $W \in \mathrm{Sb} U$ and $z \in(I \cap W) ; U ; U$. Then there are $w \in I \cap W$, $u \in U, x \in(I \cap W) ; U$, and $y \in U$ such that $\langle w, u, x\rangle,\langle x, y, z\rangle \in C$. But $x \in\{w\} ;\{u\} \subseteq I ;\{u\}=\{u\}$, so $x=u$. It follows from (d) that $\langle w, z, z\rangle \in C$, so $z \in\{w\} ;\{z\} \subseteq(I \cap W) ; U$. Thus $(I \cap W) ; U ; U \subseteq(I \cap W) ; U$. The opposite inclusion follows from 1.13(4).

(4) Assume $\mathbb{E} \mathfrak{m} \mathfrak{U} \in S A$. Then (a)-(c) hold by (2). Suppose $\langle v, w, x\rangle,\langle x, y$, $z\rangle \in C$. Then $z \in\{x\} ;\{y\} \subseteq\{v\} ;\{w\} ;\{y\} \subseteq\{v\} ; U ; U=\{v\} ; U$ by the SL, so $\langle v, u, z\rangle \in C$ for some $u \in U$. Thus (e) holds.

Now suppose $\mathfrak{U}$ satisfies (a)-(c) and (e). Then $\mathfrak{E} \mathfrak{m} \mathfrak{U} \in N A$ by (2), and $V ; U \subseteq$ $V ; U ; U$ for every $V \in \operatorname{Sb} U$ by $1.13(4)$. Suppose $z \in V ; U ; U$. Then there are $v \in V$, $w \in U, x \in V ; U$, and $y \in U$ such that $\langle v, w, x\rangle,\langle x, y, z\rangle \in C$. By (e) there is some $u \in U$ such that $\langle v, u, z\rangle \in C$, so $z \in\{v\} ;\{u\} \subseteq V ; U$. Therefore $V ; U ; U=V ; U$, and $\mathbb{m} \mathfrak{m} \in S A$.

(5) Assume $\sqrt{ } \mathfrak{M} \mathfrak{U} \in R A$. Then (a)-(c) hold by (2). Let $\langle v, w, x\rangle,\langle x, y, z\rangle \in C$. Then $z \in\{x\} ;\{y\} \subseteq\{v\} ;\{w\} ;\{y\}=\{v\} ;(\{w\} ;\{y\})$ by the AL, so there is some $u \in\{w\} ;\{y\}$ (hence $\langle w, y, u\rangle \in C$ ) such that $\langle v, u, z\rangle \in C$. Thus (f) holds. 
Suppose $\mathfrak{U}$ satisfies (a)-(c) and (f). Then $\mathfrak{E} \mathfrak{m} \mathfrak{U} \in N A$ by (2). We need only show $V ; W ; Y \subseteq V ;(W ; Y)$ for all $V, W, Y \in \mathrm{Sb} U$, since the AL follows from this by 1.16 . Let $z \in V ; W ; Y$. Then there are $v \in V, w \in W, x \in V ; W$, and $y \in Y$ such that $\langle v$, $w, x\rangle,\langle x, y, z\rangle \in C$. By (f) there is some $u \in U$ such that $\langle v, u, z\rangle,\langle w, y, u\rangle \in C$. Hence $z \in\{v\} ;\{u\} \subseteq\{v\} ;(\{w\} ;\{y\}) \subseteq V ;(W ; Y)$. Thus $V ; W ; Y \subseteq V ;(W ; Y)$.

Problem 2.3. Is there a set of first-order conditions on $\mathfrak{U}=\langle U, C, f, I\rangle$ which is equivalent to $\mathbb{E} \mathfrak{m} \mathfrak{U} \in R R A$ ? In other words is $\{\mathfrak{U}: \mathbb{E} \mathfrak{m} \mathfrak{U} \in R R A\}$ an elementary class?

Some sufficient conditions for $\mathbb{E} \mathfrak{m} \mathfrak{U} \in R R A$ are known. For example, it follows from Theorems 4.27 and 4.29 of [7] that $\mathbb{E} \mathfrak{m} \mathfrak{U} \in R R A$ whenever $\mathfrak{U}$ satisfies (a)-(c) and (f), and $I=\{w:\langle x, w, x\rangle \in C$ for some $x \in U\}$. An alternative formulation of this condition can be obtained by showing that if $\mathfrak{U}$ satisfies (a)-(c) and (f) (i.e. $(\mathfrak{F} \mathfrak{U} \in R A)$ then $I=\{w:\langle x, w, x\rangle \in C$ for some $x \in U\}$ iff $C$ is functional (i.e. if $\langle x, y, z\rangle,\langle x, y, w\rangle \in C$, then $z=w$ ). A more general sufficient (but not necessary) condition for $\mathfrak{E} \mathfrak{m} \mathfrak{U} \in R R A$ can be obtained from Theorem 7 of [11]: if $\mathfrak{U}$ satisfies (a)-(c) and (f), and for every $u \in U$ there are $p, q \in U$ such that $\langle p, u, q\rangle \in C$, and $w \in I$ whenever $\langle p, w, p\rangle \in C$ or $\langle q, w, q\rangle \in C$, then $\mathbb{\mathfrak { m }} \mathfrak{U} \in R R A$.

Definition 2.4. Let $\alpha$ be a nonzero cardinal and let $X \subseteq\{1,2,3\}$. Then

$$
\begin{gathered}
D_{\alpha}=\bigcup_{\kappa<\alpha}\{\langle 0, \kappa, \kappa\rangle,\langle\kappa, 0, \kappa\rangle,\langle\kappa, \kappa, 0\rangle\}, \\
C_{\alpha}(X)=D_{\alpha} \cup\{\langle\kappa, \lambda, \mu\rangle: 0<\kappa, \lambda, \mu<\alpha \text { and }|\{\kappa, \lambda, \mu\}| \in X\},
\end{gathered}
$$

and

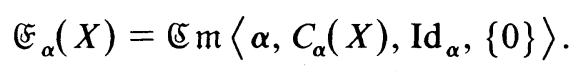

THEOREM 2.5. (1) Let $\mathfrak{U} \in N A$. Then $\mathfrak{U} \cong \&_{\alpha}(X)$ for some $\alpha>0$ and some $X \subseteq\{1$, $2,3\}$ iff the following conditions hold:

(a) $x^{\cup}=x$ for every $x \in A$,

(b) $\mathfrak{U}$ is complete and atomic,

(c) l' is an atom of $\mathfrak{A}$,

(d) if $f$ is a permutation of the atoms of $\mathfrak{A}$, and $f \mathrm{l}^{\prime}=1$ ', then $f$ can be extended to an automorphism of $\mathfrak{A}$.

(2) $\mho_{\alpha}(X)=\mathbb{\mho}_{\beta}(Y)$ iff one of the following conditions holds:

(a) $1=\alpha=\beta$,

(b) $2=\alpha=\beta$ and $\{1\} \cap X=\{1\} \cap Y$,

(c) $3=\alpha=\beta$ and $\{1,2\} \cap X=\{1,2\} \cap Y$,

(d) $3<\alpha=\beta$ and $X=Y$.

(3) $\mho_{\alpha}(X) \in W A \sim S A$ iff one of the following conditions holds:

(a) $3=\alpha$ and $X$ is $\{3\}$ or $\{1,3\}$,

(b) $3<\alpha$ and $X$ is $\varnothing,\{1\},\{3\}$, or $\{1,3\}$.

(4) $\mathbb{F}_{\alpha}(X) \in S A \sim R A$ iff one of the following conditions holds:

(a) $4=\alpha$ and $X=\{1,3\}$,

(b) $4<\alpha$ and $X=\{3\}$.

(5) $\varpi_{\alpha}(X) \in R A$ in all cases not listed in (3) or (4). 
Proof. (1) and (2) are fairly obvious. For (3)-(5) use 2.2.

COROllary 2.6. $R A \subset S A \subset W A$.

Algebras which show $W A \subset N A$ will be constructed later. It is well known that $R R A \subset R A$. (This was first proved in [8].) For some values of $\alpha$ and $X$, $\mathbb{F}_{\alpha}(X)$ is a nonrepresentable $R A$. For example, if $4 \leqslant \alpha$, then $\mho_{\alpha}(\{2\}) \in R A \sim R R A$ (see [10] or $\left[12\right.$, p. 63]), and it follows from [9] that $\mathbb{F}_{\alpha}(\{1,3\}) \in R A \sim R R A$ iff there is no projective plane of order $\alpha-2$. The Bruck-Ryser Theorem, on the nonexistence of projective planes, implies that $E_{\alpha}(\{1,3\}) \in R A \sim R R A$ for infinitely many values of $\alpha$. These nonrepresentable $R A$ 's are used in [14] to show that $R R A$ is not finitely axiomatizable. In the other direction, we have $\mathbb{E}_{\alpha}(X) \in R R A$ whenever $\mathbb{F}_{\alpha}(X) \in R A$ and $\alpha \leqslant 3, \mathbb{F}_{4}(\{3\}) \in R R A$, and $\mathbb{F}_{\alpha}(\{1,2\}), \mathbb{F}_{\alpha}(\{1,2,3\}) \in R R A$ for all $\alpha$. (See [12].) The remaining unsettled cases are collected in the following problem.

Problem 2.7. For what values of $\alpha$ is $\mho_{\alpha}(\{2,3\}) \in R R A$ or $\mho_{\alpha}(\{1,3\}) \in R R A$ ?

\section{Atom structures.}

Definition 3.1. Suppose $\mathfrak{A}$ is a relation-type algebra such that $\mathfrak{B} \mathfrak{l} \mathfrak{A} \in B A$. Let At $\mathfrak{A}$ be the set of atoms of $\mathfrak{A}$, i.e. At $\mathfrak{A}=\{x: 0 \neq x \in A$ and for every $y \in A$, either $x \cdot y=0$ or $x \cdot y=x\}$. We say that ${ }^{\cup}$ preserves atoms in $\mathfrak{A}$ if $x^{\cup} \in$ At $\mathfrak{A}$ whenever $x \in$ At $\mathfrak{A}$.

Definition 3.2. Suppose $\mathfrak{A}$ is a relation-type algebra, $\mathfrak{B} \mathfrak{I} \mathfrak{A} \in B A$, and $\cup$ preserves atoms in $\mathfrak{A}$. Then $\mathfrak{A} t \mathfrak{A}=\left\langle\right.$ At $\left.\mathfrak{A}, C^{(\mathfrak{A})}, \cup, I^{(\mathfrak{A})}\right\rangle$ is the atom structure of $\mathfrak{A}$, where $C^{(\mathfrak{U})}=\{\langle x, y, z\rangle: x, y, z \in$ At $\mathfrak{A}$ and $x ; y \geqslant z\}$ and $I^{(\mathfrak{A})}=\left\{x: 1^{\prime} \geqslant x \in\right.$ At $\left.\mathfrak{A}\right\}$. The triples in $C^{(\mathfrak{A})}$ are the cycles of $\mathfrak{A}$, and the elements of $I^{(\mathfrak{U})}$ are the identity atoms of $\mathfrak{A}$.

Theorem 3.3. Let $\mathfrak{U}=\langle U, C, f, I\rangle$ where $C \subseteq{ }^{3} U, f \in U U$, and $I \subseteq U$. Then $\cup$ preserves atoms in $\sqrt{ } \mathfrak{m} \mathfrak{U}$ and $\mathfrak{A} t \mathfrak{E} \mathfrak{m} \mathfrak{U} \cong \mathfrak{U}$. In fact, $\langle\{x\}: x \in U\rangle$ is an isomorphism from $\mathfrak{U}$ onto $\mathfrak{A} t \mathfrak{C} \mathfrak{m} \mathfrak{U}$.

ThEOREM 3.4. If $\mathfrak{A} \in N A$, then $\cup$ preserves atoms in $\mathfrak{A}$.

Proof. Let $x \in$ At $\mathfrak{A}$. Then for every $y \in A$, either $x \cdot y^{\cup}=0$ or $x \cdot y^{\cup}=x$. But $x \cdot y^{\cup}=0$ iff $x^{\cup} \cdot y=0$ by 1.11, and $x \cdot y^{\cup}=x$ iff $x^{\cup} \cdot y=x^{\cup}$ by 1.13(11), (8). Hence $x^{\cup} \cdot y=0$ or $x^{\cup} \cdot y=x^{\cup}$. Also, $0 \neq x^{\cup}$ since otherwise $0=0^{\cup}=x^{\cup \cup}=x$ by 1.13(7), (8). Thus $x^{\cup} \in$ At $\mathfrak{A}$.

The notion of atom structure was introduced as a way of recapturing the structure from which a complex algebra is constructed, as in 3.3. (A similar situation occurs in [4, pp. 453-461].) But this construction can be applied to any $N A$, as shown by 3.4 , so we may consider the algebra $\mathfrak{E} \mathfrak{m} \mathfrak{A} \mathfrak{t} \mathfrak{A}$ obtained from an arbitrary $\mathfrak{A} \in N A$. We shall see that $\mathfrak{E} \mathfrak{m} \mathfrak{A} \mathfrak{t} \mathfrak{A} \cong \mathfrak{A}$ whenever $\mathfrak{A}$ is complete and atomic, but $\mathfrak{5} \mathfrak{m} \mathfrak{A} \mathfrak{t} \mathfrak{A} \notin N A$ for some complete nonatomic $\mathfrak{A} \in N A$. On the other hand, $\sqrt{\mathfrak{m}} \mathfrak{A} \mathfrak{A} \mathfrak{A} \in W A$ whenever $\mathfrak{A} \in W A$. To prove the latter result we need a theorem about atoms in $W A$ 's which fails for $N A$ 's.

Theorem 3.5. Let $\mathfrak{A} \in W A$. If $x \in$ At $\mathfrak{A}$, then $x ; x^{\cup} \cdot 1^{\prime}, x^{\cup} ; x \cdot 1^{\prime} \in$ At $\mathfrak{A}$. 
Proof. Let $x \in$ At $\mathfrak{A}$. We first show $x ; x^{U} \cdot 1^{\prime} \in$ At $\mathfrak{A}$. We have $0 \neq x=1 ' ; x \cdot x$ by the IL, so $0 \neq x ; x^{\cup} \cdot 1^{\prime}$ by the RPL. Let $y \in A$, and suppose $x ; x^{\cup} \cdot 1^{\prime} \cdot y \neq 0$. Then $\left(1^{\prime} \cdot y\right) ; x \cdot x \neq 0$ by the RPL, and hence $x \leqslant\left(1^{\prime} \cdot y\right) ; x$. Consequently

$$
\begin{aligned}
x ; x^{\cup} \cdot 1^{\prime} & \leqslant\left(1^{\prime} \cdot y\right) ; x ; x^{\cup} \cdot 1^{\prime} & & 1.13(3) \\
& \leqslant\left(1^{\prime} \cdot y\right) ; 1 ; 1 \cdot 1, & & 1.13(3) \\
& =\left(1^{\prime} \cdot y\right) ; 1 \cdot 1^{\prime}, & & \mathrm{WL} \\
& \leqslant\left(1^{\prime} \cdot y\right) ;\left(1 \cdot\left(1^{\prime} \cdot y\right)^{\cup} ; 1^{\prime}\right) & & 1.4(1) \\
& \leqslant y ;\left(1^{\prime} ; 1^{\prime}\right) & & 1.13(3), \\
& =y & & 1.13(14)
\end{aligned}
$$

so $x ; x^{\cup} \cdot 1^{\prime} \cdot y=x ; x^{\cup} \cdot 1^{\prime}$. Thus $x ; x^{\cup} \cdot 1^{\prime} \in$ At $\mathfrak{A}$. One can prove $x^{\cup} ; x \cdot 1^{\prime} \in$ At $\mathfrak{A}$ similarly, or notice that $x^{\cup} ; x \cdot 1^{\prime}=\left(x ; x^{\cup} \cdot 1^{\prime}\right)^{\cup}$ by 1.13(8), (11), (13), (16) and use 3.4 , or apply 1.16 .

Definition 3.6. $\mathfrak{N}=\sqrt{ } \mathrm{m}\left\langle\{0,1,2\}, C, \mathrm{Id}_{3}, 1^{\prime}\right\rangle$ where $1^{\prime}=\{0,1\}$ and $C=\{\langle 0,0$, $0\rangle,\langle 1,1,1\rangle,\langle 0,2,2\rangle,\langle 2,0,2\rangle,\langle 2,2,0\rangle,\langle 1,2,2\rangle,\langle 2,1,2\rangle,\langle 2,2,1\rangle\}$.

TheOREM 3.7. (1) $\mathfrak{R} \in N A \sim W A$ and $W A \subset N A$.

(2) Theorem 3.5 fails in $\mathfrak{R}$.

Proof. It is easy to check that $\mathfrak{R}$ is the complex algebra of a structure satisfying 2.2(a)-(c), so $\mathfrak{N} \in N A$. $\mathfrak{N} \notin W A$ since $\{0\} ;\{0,1,2\}=\{0,2\}$ but $\{0\} ;\{0,1,2\}$; $\{0,1,2\}=\{0,2\} ;\{0,1,2\}=\{0,1,2\}$. Theorem 3.5 fails since $\{2\} \in$ At $\mathfrak{N}$ but $\{2\}$; $\{2\}^{\cup} \cap 1^{\prime}=1^{\prime} \notin$ At $\mathfrak{R}$.

Theorem 3.8. If $\mathfrak{A} \in W A$, then $\mathfrak{E} \mathfrak{m} \mathfrak{A} t \mathfrak{A} \in W A$.

Proof. It suffices to show $\mathfrak{U} \mathfrak{t} \mathfrak{A}$ satisfies 2.2(a)-(d). Let $\langle x, y, z\rangle \in C^{(\mathfrak{A})}$. Then $x ; y \geqslant z$, so $x ; y \cdot z \neq 0$. We get $x^{\cup} ; z \cdot y \neq 0$ and $z ; y^{\cup} \cdot x \neq 0$ by the Peircean laws, so $x^{\cup} ; z \geqslant y$ and $z ; y^{\cup} \geqslant x$ since $x, y \in$ At $\mathfrak{A}$. Hence $\left\langle x^{\cup}, z, y\right\rangle,\left\langle z, y^{\cup}, x\right\rangle \in C^{(\mathfrak{A})}$. Thus $\mathfrak{A} \mathfrak{t} \mathfrak{A}$ satisfies 2.2(a), (b).

If $w \in I^{(\mathfrak{A})}$ and $\langle x, w, y\rangle \in C^{(\mathfrak{A})}$, then $y \leqslant x ; w \leqslant x ; 1^{\prime}=x$, so $y=x$ since $x$, $y \in$ At $\mathfrak{A}$. On the other hand, for any $x \in$ At $\mathfrak{A}$ we have $x^{\cup} ; x \cdot 1$ ' $\in$ At $\mathfrak{A}$ by 3.5 , and $\left\langle x, x^{\cup} ; x \cdot 1^{\prime}, x\right\rangle \in C^{(\mathfrak{A})}$ since $x=x ; 1^{\prime} \cdot x \leqslant x ;\left(1^{\prime} \cdot x^{\cup} ; x\right)$ by the IL and $1.4(1)$. Thus 2.2(c) holds.

Suppose $w \in I^{(\mathfrak{H})}$ and $\langle w, x, x\rangle,\langle x, y, z\rangle \in C^{(\mathfrak{H})}$. Then $w \leqslant 1, x \leqslant w ; x$, and $z \leqslant x ; y$, so $z \leqslant w ; x ; y \leqslant w ; 1 ; 1=w ; 1$ by $1.13(3)$ and the WL. Consequently $z=$ $w ; 1 \cdot z \leqslant w ;\left(1 \cdot w^{\cup} ; z\right) \leqslant w ;(1 \cup ; z)=w ; z$ by $1.4(1), 1.13(3),(12),(14)$, and the IL. Thus $\langle w, z, z\rangle \in C^{(\mathfrak{A})}$, so $\mathfrak{A} \mathrm{t} \mathfrak{A}$ satisfies $2.2(\mathrm{~d})$.

THEOREM 3.9. There is a complete $\mathfrak{A} \in N A$ such that $\mathbb{E} \mathfrak{m} \mathfrak{A} \mathfrak{t} \mathfrak{A} \notin N A$.

Proof. Let $B=\langle B,+, \cdot,-, 0,1\rangle$ be the direct product of a complete atomless $B A$ (e.g. $\S 20(C)$ of $[16])$ and a 2-element $B A$. Then $\mathfrak{B}$ is a complete infinite $B A$ with 
exactly one atom. Let $0^{\prime}$ be the unique atom of $\mathscr{B}$ and let $1^{\prime}=-0$ '. Define ; on $B \times B$ as follows:

$$
x ; y= \begin{cases}0 & \text { if } x=0 \text { or } y=0 \\ x \cdot y & \text { if } 0 \neq x \leqslant 1 \text { and } 0 \neq y \leqslant 1 \\ x \cdot y+0, & \text { if } x \geqslant 0 \text {, and } 0 \neq y \leqslant 1 \\ x \cdot y+0, & \text { if } 0 \neq x \leqslant 1 \text { ' and } y \geqslant 0 \\ 1 & \text { if } x \geqslant 0\end{cases}
$$

Set $\mathfrak{A}=\left\langle B,+, \cdot,-, 0,1, ; \mathbb{I d}_{B}, 1^{\prime}\right\rangle$. Notice that ; is commutative. We shall see that $\mathfrak{A} \in N A$ but the IL fails in $\mathbb{E} \mathfrak{m} \mathfrak{A} \mathfrak{t} \mathfrak{A}$.

The verification of the IL in $\mathfrak{A}$ splits into three cases: if $x=0$, then $x ; 1^{\prime}=1^{\prime} ; x=$ $0=x$; if $0 \neq x \leqslant 1^{\prime}$, then $x ; 1^{\prime}=1^{\prime} ; x=1^{\prime} \cdot x=x$; finally, if $x \geqslant 0$ ', then $x ; 1^{\prime}=1^{\prime} ; x$ $=x \cdot 1^{\prime}+0^{\prime}=x \cdot 1^{\prime}+x \cdot 0^{\prime}=x \cdot\left(1^{\prime}+0^{\prime}\right)=x \cdot 1=x$.

Let $x, y, z \in B$ and set $a=x ; y \cdot z, b=x ; z \cdot y$, and $c=z ; y \cdot x$. To show $\mathfrak{A}$ satisfies the Peircean laws it suffices, since converse is the identity in $\mathfrak{A}$, to show $a=0$ iff $b=0$ iff $c=0$. If $0 \in\{x, y, z\}$, then $a=b=c=0$, so assume $0 \neq x, y, z$. The following facts are easy to check. If $x+y \leqslant 1$ ' or $x+z \leqslant 1$ ' or $y+z \leqslant 1$ ', then $a=b=c$. If $0^{\prime} \leqslant x \cdot y$ and $z \leqslant 1^{\prime}$, then $a=z \neq 0$ and $0^{\prime} \leqslant b=c$. Similarly for permutations of $x, y$, and $z$. Finally, if $0^{\prime} \leqslant x \cdot y \cdot z$, then $a=x \neq 0, b=y \neq 0$, and $c=z \neq 0$. This exhausts the possibilities, and in each case $a=0$ iff $b=0$ iff $c=0$. Thus $\mathfrak{A} \in N A$.

However, At $\mathfrak{A}=\left\{0^{\prime}\right\}, C^{(\mathfrak{A})}=\left\{\left\langle 0^{\prime}, 0^{\prime}, 0^{\prime}\right\rangle\right\}$, and $I^{(\mathfrak{A})}=\varnothing$, so $\sqrt{ } \mathfrak{m} \mathfrak{A} \mathfrak{t} \mathfrak{A} \cong$ $\left\langle\{0,1\},+, \cdot,-, 0,1, \cdot, \operatorname{Id}_{\{0,1\}}, 0\right\rangle$. The IL fails since $1 ; 0=1 \cdot 0=0 \neq 1$, so (5) $\mathfrak{A} \mathrm{t} \mathfrak{A} \notin N A$.

Theorem 3.9 shows that the hypothesis of 3.8 cannot be weakened from $\mathfrak{A} \in W A$ to $\mathfrak{A} \in N A$. The next theorem shows that no stronger conclusion than $\mathfrak{E} \mathfrak{m} \mathfrak{A} \mathfrak{t} \mathfrak{A} \in$ $W A$ can be obtained by assuming $\mathfrak{A} \in R R A$.

Theorem 3.10. There is some $\mathfrak{A} \in R R A$ such that $\mathbb{E} \mathfrak{m} \mathfrak{A} t \mathfrak{A} \in W A \sim S A$.

Proof. Let $U=\left\{e_{00}, e_{11}, e_{22}, d_{11}, d_{22}, d_{01}, d_{10}, d_{02}, d_{20}\right\}, U^{\prime}=U \cup\left\{d_{12}^{r}: r \in Q\right\}$ $\cup\left\{d_{21}^{r}: r \in Q\right\}$ (where $Q$ is the set of rational numbers), and $I=\left\{e_{00}, e_{11}, e_{22}\right\}$. Define $f$ on $U^{\prime}$ as follows, for all $r \in Q$ :

\begin{tabular}{c|c|c|c|c|c|c|c|c|c|c|c}
$x$ & $e_{00}$ & $e_{11}$ & $e_{22}$ & $d_{11}$ & $d_{22}$ & $d_{01}$ & $d_{10}$ & $d_{02}$ & $d_{20}$ & $d_{12}^{r}$ & $d_{21}^{r}$ \\
\hline$f x$ & $e_{00}$ & $e_{11}$ & $e_{22}$ & $d_{11}$ & $d_{22}$ & $d_{10}$ & $d_{01}$ & $d_{20}$ & $d_{02}$ & $d_{21}^{r}$ & $d_{12}^{r}$
\end{tabular}

Notice that $U$ is closed under $f$. If $\langle x, y, z\rangle \in{ }^{3} U^{\prime}$ then the transforms of $\langle x, y, z\rangle$ are $\langle x, y, z\rangle,\langle f x, z, y\rangle,\langle y, f z, f x\rangle,\langle f y, f x, f z\rangle,\langle f z, x, f y\rangle$, and $\langle z, f y, x\rangle$. Let $C^{\prime}$ be the set of transforms of the following triples, for all $r, s \in Q$ :

$$
\begin{array}{lll}
\left\langle e_{11}, e_{11}, e_{11}\right\rangle, & \left\langle e_{22}, e_{22}, e_{22}\right\rangle, & \left\langle e_{00}, e_{00}, e_{00}\right\rangle, \\
\left\langle d_{11}, e_{11}, d_{11}\right\rangle, & \left\langle d_{22}, e_{22}, d_{22}\right\rangle, & \left\langle d_{10}, e_{00}, d_{10}\right\rangle, \\
\left\langle d_{01}, e_{11}, d_{01}\right\rangle, & \left\langle d_{02}, e_{22}, d_{02}\right\rangle, & \left\langle d_{20}, e_{00}, d_{20}\right\rangle, \\
\left\langle d_{21}^{r}, e_{11}, d_{21}^{r}\right\rangle, & \left\langle d_{12}^{r}, e_{22}, d_{12}^{r}\right\rangle, &
\end{array}
$$




$$
\begin{array}{lll}
\left\langle d_{11}, d_{11}, d_{11}\right\rangle, & \left\langle d_{22}, d_{22}, d_{22}\right\rangle, & \left\langle d_{01}, d_{12}^{r}, d_{02}\right\rangle, \\
\left\langle d_{01}, d_{11}, d_{01}\right\rangle, & \left\langle d_{02}, d_{22}, d_{02}\right\rangle, & \\
\left\langle d_{21}^{r}, d_{11}, d_{21}^{s}\right\rangle, & \left\langle d_{12}^{r}, d_{22}, d_{12}^{s}\right\rangle .
\end{array}
$$

Let $C=C^{\prime} \cap^{3} U$. Let $\mathfrak{B}=\mathfrak{c} \mathfrak{m}\langle U, C, f, I\rangle$ and $\mathfrak{c}=\sqrt{\mathfrak{m}}\left\langle U^{\prime}, C^{\prime}, f, I\right\rangle$. We will show:

(1) $\mathfrak{B} \in W A \sim S A$,

(2) $\mathbb{E} \in R R A$,

(3) for some $\mathfrak{A} \subseteq \mathfrak{E}, \mathfrak{C} \mathfrak{m} \mathfrak{A} t \mathfrak{U} \cong \mathfrak{B}$.

If (1)-(3) hold, then the proof is complete, since $\mathfrak{A} \in R R A$ by (2) and (3), but (5) $\mathfrak{m} \mathfrak{A} \mathfrak{A} \in W A \sim S A$ by (1) and (3).

It is clear that $\langle U, C, f, I\rangle$ satisfies $2.2(\mathrm{a})-(\mathrm{c})$, and it is easy to show that it also satisfies 2.2(d), so $\mathfrak{B} \in W A$. On the other hand, $\mathfrak{B} \notin S A$ since $\langle U, C, f, I\rangle$ does not satisfy $2.2(\mathrm{e}):\left\langle d_{01}, d_{10}, e_{00}\right\rangle,\left\langle e_{00}, d_{02}, d_{02}\right\rangle \in C$ but there is no $x \in U$ such that $\left\langle d_{01}, x, d_{02}\right\rangle \in C$.

To show $\mathbb{E} \in R R A$ we need two countable infinite disjoint sets $K$ and $L$, an element $m \notin K \cup L$, and a function $p$ : $(K \times L) \cup(L \times K) \rightarrow Q$ which has the following properties:

(4) $p(k, l)=p(l, k)$ for every $k \in K$ and $l \in L$,

(5) if $k_{1}, k_{2} \in K, k_{1} \neq k_{2}$, and $r, s \in Q$, then there is some $l \in L$ such that $p\left(k_{1}, l\right)=r$ and $p\left(k_{2}, l\right)=s$,

(6) if $l_{1}, l_{2} \in L, l_{1} \neq l_{2}$, and $r, s \in Q$, then there is some $k \in K$ such that $p\left(k, l_{1}\right)=r$ and $p\left(k, l_{2}\right)=s$.

Such sets and functions are easy to construct. For example, let $M_{0}=Q, M_{1}=M_{0}$ $\times Q \times M_{0} \times Q$, and $M_{n+2}=M_{n} \cup\left(M_{n+1} \times Q \times M_{n+1} \times Q\right)$ for every $n<\omega$. Set $K=\cup_{n<\omega} M_{2 n}$ and $L=\cup_{n<\omega} M_{2 n+1}$. If $\langle x, r, y, s\rangle \in M_{n} \times Q \times M_{n} \times Q$ and $x \neq y$, then set

(7) $p(x,\langle x, r, y, s\rangle)=p(\langle x, r, y, s\rangle, x)=r$,

(8) $p(y,\langle x, r, y, s\rangle)=p(\langle x, r, y, s\rangle, y)=s$, and let $p(k, l)=p(l, k)=0$ for all $k \in K, l \in L$, not covered in (7) or (8). (In fact, since (7) and (8) imply (5) and (6), $p$ can be extended to $(K \times L) \cup(L \times K)$ in any manner consistent with (4), (7), and (8).)

Suppose $K, L, m$, and $p$ satisfy (4)-(6). Then we get an embedding $F$ of $\mathbb{E}$ into $\Re e(K \cup L \cup\{m\})$ as follows:

$$
\begin{array}{lll}
F\left(e_{00}\right)=\{\langle m, m\rangle\}, & F\left(d_{01}\right)=\{m\} \times K, & F\left(e_{11}\right)=\operatorname{Id}_{K}, \\
F\left(d_{10}\right)=K \times\{m\}, & F\left(e_{22}\right)=\operatorname{Id}_{L}, & F\left(d_{02}\right)=\{m\} \times L, \\
F\left(d_{11}\right)=K \times K, & F\left(d_{20}\right)=L \times\{m\}, & F\left(d_{22}\right)=L \times L, \\
F\left(d_{12}^{r}\right)=\{\langle k, l\rangle: k \in K, l \in L, p(k, l)=r\} & \text { for all } r \in Q, \\
F\left(d_{21}^{r}\right)=\{\langle l, k\rangle: l \in L, k \in K, p(l, k)=r\} & \text { for all } r \in Q, \\
F(X)=\bigcup_{x \in X} F(x) & \text { for all } X \subseteq U^{\prime} .
\end{array}
$$

Thus $\mathbb{E} \in R R A$. Now we choose $\mathfrak{A} \subseteq \mathbb{E}$. Let $H$ be the set of all subsets of $Q$ which are finite unions of intervals $Q \cap(a, b)$ where $a$ and $b$ are irrational. Then $\langle H, \cup$, 
$\left.\cap, Q_{Q} \sim, \varnothing, Q\right\rangle$ is an atomless $B A$. Let $A$ be the set of subsets of $U^{\prime}$ of the form $X \cup\left\{d_{12}^{r}: r \in R\right\} \cup\left\{d_{21}^{s}: s \in S\right\}$ where $X \subseteq U$ and $R, S \in H$. Then it is not difficult to verify that $A$ is closed under $\cup, \cap, U^{\prime} \sim$, ; and ${ }^{\cup}$, hence is the universe of a subalgebra $\mathfrak{A}$ of $\mathfrak{E}$, and that $\mathbb{E} \mathfrak{m} \mathfrak{A} \mathfrak{t} \mathfrak{A} \cong \mathfrak{B}$.

Problem 3.11. Characterize the class $\{\mathfrak{E} \mathfrak{m} \mathfrak{A} t \mathfrak{A}: \mathfrak{A} \in R R A\}$. Does it contain every complete atomic $W A$ ? What variety does it generate?

Problem 3.12 (raised by Don Pigozzi). Suppose $K \subseteq N A, K$ is a variety, and (5) $\mathfrak{A} \mathfrak{A} \mathfrak{A} \in K$ for every $\mathfrak{A} \in K$. Is $K \subseteq W A$ ?

The algebras used in the proofs of 3.9 and 3.10 are not atomic. The next theorem shows that this is unavoidable.

THEOREM 3.13. Assume $\mathfrak{A} \in N A$ and $\mathfrak{A}$ is atomic.

(1) $\mathfrak{A}$ is isomorphic to a subalgebra of $\mathfrak{E} \mathfrak{m} \mathfrak{A} t \mathfrak{A}$.

(2) $\mathfrak{A} \cong \mathbb{S} \mathfrak{m} \mathfrak{A} \mathfrak{t} \mathfrak{A}$ iff $\mathfrak{A}$ is complete.

(3) $\mathfrak{s} \mathfrak{m} \mathfrak{U} \mathfrak{a} \in N A$.

(4) $\mathfrak{c} \mathfrak{m} \mathfrak{U} \mathfrak{t} \mathfrak{A}$ is a $W A, S A, R A$, or $R R A$ iff $\mathfrak{A}$ is a $W A, S A, R A$, or $R R A$, respectively.

Proof. (1) Define $f: A \rightarrow$ Sb At $\mathfrak{A}$ by $f x=\{a: x \geqslant a \in$ At $\mathfrak{A}\}$ for every $x \in A$. It is well known that $f$ embeds $\mathfrak{B l} \mathfrak{A}$ into $\mathfrak{B l} \mathfrak{C} \mathfrak{m} \mathfrak{A} \mathfrak{t} \mathfrak{A}$ (see [16, Theorem 9.1, p. 28]), and $x=\sum f x$ for every $x \in A$. Obviously, $f 1^{\prime}=I^{(\mathfrak{A})}$, so we need only show $f(x ; y)=f x ; f y$ and $f\left(x^{\cup}\right)=(f x)^{\cup}$ for all $x, y \in A$. To establish the first formula, note that the following formulas are equivalent for every $a \in$ At $\mathfrak{A}$.

$$
\begin{aligned}
a & \in f(z ; y), \\
a & \leqslant x ; y=\sum\{b ; c: b \in f x, c \in f y\}, \\
a & \leqslant b ; c \text { for some } b \in f x, c \in f y, \\
\langle b, c, a\rangle & \in C^{(\mathfrak{I})} \quad \text { for some } b \in f x, c \in f y, \\
a & \in f x ; f y .
\end{aligned}
$$

Another sequence of equivalent formulas, with $a \in$ At $\mathfrak{A}$, proves $f\left(x^{\cup}\right)=(f x)^{\cup}$;

$$
\begin{aligned}
& a \in f\left(x^{\cup}\right), \quad a \leqslant x^{\cup}=\left(\sum f x\right)^{\cup}=\sum_{b \in f x} b^{\cup}, \\
& a=b^{\cup} \quad \text { for some } b \in f x, \quad a \in(f x)^{\cup} .
\end{aligned}
$$

(2) If $\mathfrak{U} \cong \mathfrak{E} \mathfrak{m} \mathfrak{A} \mathfrak{A} \mathfrak{A}$, then $\mathfrak{A}$ is obviously complete. Suppose $\mathfrak{A}$ is complete. In view of (1), it suffices to show $f$ is onto. Let $X \subseteq$ At $\mathfrak{A}$. Then $\Sigma X$ exists and $f\left(\sum X\right)=X$. Thus $f$ is onto and $\mathfrak{A} \cong \mathbb{E} \mathfrak{m} \mathfrak{A} t \mathfrak{A}$.

(3) We show $\mathfrak{A} \mathfrak{t} \mathfrak{A}$ satisfies 2.2(a)-(c). The proof of 2.2(a), (b) and half of (c) is the same as the first part of the proof of 3.8. For the other half of 2.2(c) we must prove, given $x \in$ At $\mathfrak{A}$, that $\langle x, w, x\rangle \in C^{(\mathfrak{A})}$ for some $w \in I^{(\mathfrak{A})}$. This follows from $x=$ $x ; 1^{\prime}=x ; \sum I^{(\mathfrak{A})}=\Sigma\left\{x ; w: w \in I^{(\mathfrak{A})}\right\}$. Thus $\mathbb{E} \mathfrak{m} \mathfrak{A} \mathfrak{t} \mathfrak{A} \in N A$.

(4) We will prove that if $\mathfrak{U}$ is a $W A, S A$, or $R A$, then $\mathfrak{A} \downarrow \mathfrak{U}$ satisfies $2.2(\mathrm{~d})$, (e), or (f), respectively. 
Let $\mathfrak{U} \in W A, w \in I^{(\mathfrak{A})}$, and $\langle w, x, x\rangle,\langle x, y, z\rangle \in C^{(\mathfrak{A})}$. Then $z \leqslant x ; y \leqslant w ; x ; y$ $\leqslant w ; 1 ; 1=w ; 1$ by $1.13(3)$ and the WL, so $z \leqslant w ; \Sigma$ At $\mathfrak{A}=\Sigma\{w ; u: u \in$ At $\mathfrak{A}\}$. Hence $z \leqslant w ; u$ for some $u \in$ At $\mathfrak{A}$. However $z \leqslant w ; u \leqslant 1^{\prime} ; u=u$, so $z=u$, and $\langle w, z, z\rangle \in C^{(\mathfrak{A})}$. Thus 2.2(d) holds in $\mathfrak{A} t \mathfrak{A}$ and $\mathfrak{C} \mathfrak{m} \mathfrak{A} t \mathfrak{A} \in W A$.

Let $\mathfrak{A} \in S A$, and $\langle v, w, x\rangle,\langle x, y, z\rangle \in C^{(\mathfrak{U})}$. Then $z \leqslant x ; y \leqslant v ; w ; y \leqslant v ; 1 ; 1=$ $v ; 1$ by $1.13(3)$ and the SL, so $z \leqslant v ; \Sigma$ At $\mathfrak{A}=\Sigma\{v ; u: u \in$ At $\mathfrak{A}\}$. Hence $z \leqslant v ; u$ for some $u \in$ At $\mathfrak{A}$, and so $\langle v, u, z\rangle \in C^{(\mathfrak{I})}$. Thus $\mathfrak{A} t \mathfrak{A}$ satisfies $2.2(\mathrm{e})$ and $\mathfrak{E} \mathfrak{m} \mathfrak{A} t \mathfrak{A} \in$ $S A$.

Let $\mathfrak{A} \in R A$, and $\langle v, w, x\rangle,\langle x, y, z\rangle \in C^{(\mathfrak{A})}$. Then $z \leqslant z ; y \leqslant v ; w ; y=v ;(w ; y)$ by $1.13(3)$ and the AL, so $z \leqslant v ; \Sigma\{u: w ; y \geqslant u \in$ At $\mathfrak{A}\}$. Hence, for some $u \in$ At $\mathfrak{A}$, $u \leqslant w ; y$ and $z \leqslant v ; u$, so $\langle w, y, u\rangle,\langle v, u, z\rangle \in C^{(\mathfrak{A})}$. Thus $\mathfrak{A} \mathrm{t} \mathfrak{A}$ satisfies 2.2(f), and (c) $\mathfrak{m} \mathfrak{A} t \mathfrak{A} \in R A$.

Since $\mathfrak{A}$ is isomorphic to a subalgebra of $\mathfrak{夭} \mathfrak{m} \mathfrak{A} \mathrm{t} \mathfrak{A}$, it follows from the arguments above that $\mathbb{E} \mathfrak{m} \mathfrak{A} t \mathfrak{A}$ is a $W A, S A$, or $R A$ iff $\mathfrak{A}$ is a $W A, S A$, or $R A$, respectively. Certainly $\mathfrak{A} \in R R A$ if $\mathfrak{E} \mathfrak{m} \mathfrak{A} \mathfrak{t} \mathfrak{A} \in R R A$, so we need only prove $\mathfrak{c} \mathfrak{m} \mathfrak{A} \mathrm{t} \mathfrak{A} \in R R A$ whenever $\mathfrak{A} \in R R A$.

Suppose $f$ is an embedding of $\mathfrak{A}$ into $\subseteq \mathfrak{b} E$ for some equivalence relation $E$. Let $F X=\cup_{x \in X} f x$ for every $X \subseteq$ At $\mathfrak{A}$. Then it is easy to check that $F$ embeds $\mathfrak{E} \mathfrak{m} \mathfrak{A} t \mathfrak{A}$ into $\mathfrak{S} \mathfrak{b} E$, so $\mathfrak{E} \mathfrak{m} \mathfrak{A} \mathfrak{t} \mathfrak{A} \in R R A$.

Theorem 3.13 shows that every complete and atomic $N A$ is isomorphic to a complex algebra. In particular, every finite $N A$ is isomorphic to the complex algebra of its atom structure. Obviously not every $N A$ has this property, since there are incomplete $N A$ 's. However every atomic (and possibly incomplete) $N A$ is isomorphic to a subalgebra of a complex algebra, and we shall see later (in 4.3) that this conclusion can be extended to all $N A$ 's.

4. Perfect extensions. The notion of perfect extension is defined for $B A$ 's with operators in Definition 2.14 of [6], and applies to $N A$ 's by 1.12 .

THEOREM 4.1. Let $\mathfrak{A}, \mathfrak{B} \in N A$. Then $\mathfrak{A}$ is a perfect extension of $\mathfrak{B}$ iff the following conditions are satisfied:

(1) $\mathfrak{B} \subseteq \mathfrak{A}$,

(2) $\mathfrak{A}$ is complete and atomic,

(3) if $X \subseteq B$ and $\Sigma^{(\mathfrak{I})} X=1$, then $\Sigma^{(\mathfrak{U})} Y=1$ for some finite $Y \subseteq X$,

(4) if $a, b \in$ At $\mathfrak{A}$ then $a ; b=\Pi^{(\mathfrak{A})}\{x ; y: a \leqslant x \in B, b \leqslant y \in B\}$.

Proof. It follows from Definition 2.14 of [6] that $\mathfrak{A}$ is a perfect extension of $\mathfrak{B}$ iff (1)-(4) hold and

(5) if $a, b \in$ At $\mathfrak{A}$ and $a \neq b$, then there is some $x \in B$ such that $a \leqslant x$ and $b \cdot x=0$,

(6) if $a \in$ At $\mathfrak{A}$, then $a^{\cup}=\Pi^{(\mathfrak{A})}\left\{x^{\cup}: a \leqslant x \in B\right\}$.

We therefore need only show that (5) and (6) are consequences of (1)-(4).

Let $a \in$ At $\mathfrak{A}$. We will use (4) to show $a=\Pi^{(\mathfrak{A})}\{z: a \leqslant z \in B\}$. By 3.12, $\mathfrak{C}_{\mathfrak{m}} \mathfrak{A} t \mathfrak{A}$ $\cong \mathfrak{A} \in N A$, so $\mathfrak{A} \mathrm{t} \mathfrak{A}$ satisfies 2.2(c). Hence $\langle a, e, a\rangle \in C^{(\mathfrak{A})}$ for some $e \in I^{(\mathfrak{A})}$. Then 
$a \leqslant a ; e \leqslant a ; 1^{\prime}=a$, so $a=a ; e=\Pi^{(\mathfrak{A})}\{x ; y: a \leqslant x \in B, e \leqslant y \in B\}$ by (4). However, $\{x ; y: a \leqslant x \in B, e \leqslant y \in B\} \subseteq\{z: a \leqslant z \in B\}$, for if $a \leqslant x \in B$ and $e \leqslant y \in$ $B$, then $a=a ; e \leqslant x ; y \in B$. Consequently

$$
a \leqslant \prod^{(\mathscr{U})}\{z: a \leqslant z \in B\} \leqslant \prod^{(\mathfrak{L})}\{x ; y: a \leqslant x \in B, e \leqslant y \in B\}=a,
$$

so $a=\Pi^{(\mathscr{R})}\{z: a \leqslant z \in B\}$. Using this equation and 1.11 we have

$$
a^{\cup}=\left(\prod^{(\mathscr{U})}\{a \leqslant z \in B\}\right)^{\cup}=\prod^{(\mathscr{U})}\left\{z^{\cup}: a \leqslant z \in B\right\},
$$

so (6) holds. Suppose $b \in$ At $\mathfrak{A}$ and $b \neq a$. Then $0=b \cdot a=\Pi^{(\mathfrak{A})}\{b \cdot z: a \leqslant z \in B\}$, so $b \cdot z=0$ for some $z \in B$ such that $a \leqslant z$, i.e. (5) holds.

THEOREM 4.2. Let $\mathfrak{B} \in N A$. Then there is some $\mathfrak{A} \in N A$ such that

(1) $\mathfrak{A}$ is a perfect extension of $\mathfrak{B}$,

(2) if $\mathfrak{U}^{\prime}$ is a perfect extension of $\mathfrak{B}$, then there is a unique isomorphism $h$ of $\mathfrak{A}^{\prime}$ onto II such that $\operatorname{Id}_{B}=B 1 h$,

(3) $\mathfrak{A}$ is a WA, SA, or $R A$ iff $\mathfrak{B}$ is a WA, $S A$, or $R A$, respectively.

Proof. Theorem 2.15 of [6] insures that there is a $B A$ with operators $\mathfrak{A}$ which is a perfect extension of $\mathfrak{B}$, and 2.17 of [6] insures that $\mathfrak{A}$ is unique in the sense of (2). We wish to use 2.18 of [6] to show that $\mathfrak{A} \in N A$ and (3) holds. Since complementation is not taken as a fundamental operation of $B A$ 's in [6], the term "equationally definable class" in 2.18 refers to a class of $B A$ 's with operators which is definable by equations containing no operation symbol for complementation. It follows from 1.4 that $N A, W A, S A$, and $R A$ are such classes, so 2.18 applies.

It is not known whether $R R A$ can be defined by equations without using complementation. Nevertheless, D. Monk proved that perfect extensions of representable $R A$ 's are representable (as reported in [12, p. 66]).

THEOREM 4.3. Let $\mathfrak{A} \in N A$. Then there is a structure $\mathfrak{U}=\langle U, C, f, I\rangle$, where $C \subseteq$ ${ }^{3} U, f \in{ }^{U} U$, and $I \subseteq U$, such that

(1) $\mathfrak{u}$ satisfies $2.2(a)-(c)$, i.e. $\mathbb{E} \mathfrak{m} \mathfrak{U} \in N A$,

(2) $\mathfrak{A}$ is isomorphic to a subalgebra of $\mathfrak{E} \mathfrak{m} \mathfrak{H}$,

(3) $\mathfrak{A} \cong \mathfrak{E} \mathfrak{m} \mathfrak{U}$ iff $\mathfrak{A}$ is complete and atomic,

(4) $\mathfrak{U}$ satisfies 2.2(d), (e), or (f) iff $\mathfrak{U}$ is a WA, SA, or RA, respectively.

Proof. If $\mathfrak{A}$ is atomic, let $\mathfrak{U}=\mathfrak{A} \mathfrak{t} \mathfrak{A}$ and apply 3.13 and 2.2. Otherwise, let $\mathfrak{U}=\mathfrak{A} \mathfrak{t} \mathfrak{B}$ where $\mathfrak{B}$ is a perfect extension of $\mathfrak{A}$. Then $\mathfrak{B} \cong \mathfrak{C} \mathfrak{m} \mathfrak{U}$ by 3.13 , and (1)-(3) follow from 2.2 and 4.2 .

Theorem 4.3 may be regarded as a representation theorem, even though there are nonrepresentable $N A$ 's. The analogous theorem for cylindric algebras is 2.7.43 of [4], and the comments that follow, in Remarks 2.7 .46 , also apply to 4.3 , with certain obvious changes.

5. Relativization. In this section we consider a generalization of the notion of relativization from $B A$ 's to $N A$ 's. A similar (but different) construction is introduced 
in Definition 4.8 of [7]. The analogous construction for cylindric algebras is studied in $\$ 2.2$ of [4].

Definition 5.1. Let $\mathfrak{U} \in N A$ and $x \in A$. Then $\mathrm{R}_{x} \mathfrak{A}=\{y: x \geqslant y \in A\}$ and $\mathfrak{R l} \mathfrak{l}_{x} \mathfrak{A}=\left\langle\mathrm{Rl}_{x} \mathfrak{U},+, \cdot{ }_{x}-, 0, x, ;^{x},{ }^{x}, 1_{x}\right\rangle$, where ${ }_{x}-y=-y \cdot x, y ;{ }^{x} z=y ; z \cdot x$, and $y^{\cup x}=y^{\cup} \cdot x$ for all $y, z \in \mathrm{R}_{x} \mathfrak{A}$. $\mathfrak{R} \mathfrak{l}_{x} \mathfrak{A}$ is called the relativization of $\mathfrak{A}$ to $x$.

Some elementary consequences of this definition are given in the next two theorems.

Theorem 5.2. Let $\mathfrak{A}, \mathfrak{B} \in N A$ and $x, y \in A$.

(1) $\mathfrak{A} \mathfrak{l}_{1} \mathfrak{A}=\mathfrak{U}$.

(2) If $x \leqslant y$ then $\mathfrak{R} \mathfrak{l}_{x} \mathfrak{R} \mathfrak{l}_{y} \mathfrak{A}=\mathfrak{R} \mathfrak{l}_{x} \mathfrak{A}$.

(3) If $\mathfrak{A} \subseteq \mathfrak{B}$ then $\mathfrak{R} \mathfrak{l}_{x} \mathfrak{A} \subseteq \mathfrak{R} \mathfrak{l}_{x} \mathfrak{B}$.

THEOREM 5.3. Let $E$ be an equivalence relation.

(1) If $E \subseteq U \times U$ then $\subseteq \mathfrak{b} E=\Re \mathfrak{l}_{E} \Re$ e $U$.

(2) If $U \in(\mathrm{Fd} E) / E$ then $\Re$ e $U=\Re \mathfrak{I}_{U \times U}$ Sb $E$.

Suppose $\mathfrak{A} \in N A$ and $x \in A$. What conditions on $x$ are equivalent to $\mathfrak{A} \mathfrak{I}_{x} \mathfrak{A} \in N A$ ? The answer is given in 5.5 and motivates the following definition.

Definition 5.4. Let $\mathfrak{A} \in N A$. Then $\operatorname{Sr} \mathfrak{A}=\left\{x: x \in A\right.$ and $\left.x^{\cup}=x=\left(1^{\prime} \cdot x\right) ; x\right\}$. We call $x$ a symmetric-reflexive element of $\mathfrak{A}$ iff $x \in \operatorname{Sr} \mathfrak{A}$.

Suppose $E$ is an equivalence relation and $R \subseteq E$. Then $R \in \operatorname{Sr} \subseteq \mathfrak{b} E$ iff $R$ is symmetric $\left(R^{-1}=R\right)$ and reflexive over its field $\left(\operatorname{Id}_{\mathrm{Fd} R} \subseteq R\right)$.

Theorem 5.5. Let $\mathfrak{A} \in N A$ and $x \in A$. Then $\mathfrak{R} \mathfrak{l}_{x} \mathfrak{A} \in N A$ iff $x \in \operatorname{Sr} \mathfrak{A}$.

Proof. Let $\mathfrak{R} \mathfrak{l}_{x} \mathfrak{H} \in N A$. Then $x^{\cup x}=x$ by 1.13(9), so $x=x^{\cup} \cdot x$. Using 1.13(8), (11) we get $x^{\cup}=\left(x^{\cup} \cdot x\right)^{\cup}=x^{\cup \cup} \cdot x^{\cup}=x \cdot x^{\cup}=x$. Also $x=1{ }_{x}^{\prime} ; x$ by the IL, so $x=\left(1^{\prime} \cdot x\right) ; x \cdot x \leqslant\left(1^{\prime} \cdot x\right) ; x \leqslant 1 ; x=x$ by $1.13(3)$ and the IL. Thus $x^{\cup}=x=$ $\left(1^{\prime} \cdot x\right) ; x$, so $x \in \operatorname{Sr} \mathfrak{A}$.

Assume $x \in \operatorname{Sr} \mathfrak{A}$. Notice that $y^{\cup x}=y^{\cup}$ whenever $y \in \mathrm{R}_{x} \mathfrak{A}$, since $y^{\cup x}=y^{\cup} \cdot x$ $=y^{\cup} \cdot x^{\cup}=(y \cdot x)^{\cup}=y^{\cup}$, and $w ;{ }^{x} y \cdot z=w ; y \cdot z$ whenever $z \in \mathrm{Rl}_{x} \mathfrak{A}$. Consequently the Peircean laws for $\Re \mathfrak{l}_{x} \mathfrak{A}$ follow immediately from the Peircean laws for $\mathfrak{A}$. For the IL in $\mathfrak{R} \mathfrak{l}_{x} \mathfrak{A}$, we first note that $1_{x}^{\prime} ;{ }^{x} y=\left(1^{\prime} \cdot x\right) ; y \cdot x \leqslant 1 ; y=y$, for $y \in \mathrm{Rl}_{x} \mathfrak{A}$. Then we get

$$
\begin{array}{rlrl}
y & =y \cdot x=y \cdot\left(1^{\prime} \cdot x\right) ; x \cdot x & \\
& \leqslant\left(1^{\prime} \cdot x\right) ;\left(x \cdot\left(1^{\prime} \cdot x\right)^{\cup} ; y\right) \cdot x & & 1.4(1) \\
& \leqslant\left(1^{\prime} \cdot x\right) ;\left(1^{\cup} ; y\right) \cdot x & & 1.13(3),(12) \\
& =1_{x}^{\prime} ;{ }^{x} y & & 1.13(14), \text { IL. }
\end{array}
$$

Thus $y=1_{x} ;{ }^{x} y$ for every $y \in \mathrm{R}_{x} \mathfrak{A}$. Now if $y \in \mathrm{R}_{x} \mathfrak{A}$ then $y^{\cup} \in \mathrm{R}_{x} \mathfrak{A}$ since $y^{\cup} \leqslant x^{\cup}=x$ by $1.13(12)$, so $y^{\cup}=11_{x}^{\prime} ;{ }^{x} y^{\cup}$. Then

$$
\begin{aligned}
y & =y^{\cup \cup}=\left(1_{x}, x^{x} y^{\cup}\right)^{\cup}=\left(\left(1^{\prime} \cdot x\right) ; y^{\cup} \cdot x\right)^{\cup} \\
& =y ;\left(1^{\prime} \cdot x^{\cup}\right) \cdot x^{\cup}=y ;\left(1^{\prime} \cdot x\right) \cdot x=y ;{ }^{x} 1_{x}
\end{aligned}
$$

by 1.13(8), (11), (13), (14). Thus the IL holds in $\mathfrak{R} \mathfrak{l}_{x} \mathfrak{A}$, and $\mathfrak{R} \mathfrak{l}_{x} \mathfrak{A} \in N A$. 
Definition 5.6. If $K \subseteq N A$, then $\mathrm{Rl} K=\left\{\mathfrak{R} \mathfrak{l}_{x} \mathfrak{A}: \mathfrak{A} \in K\right.$ and $\left.x \in \operatorname{Sr} \mathfrak{A}\right\}$.

THEOREM 5.7. Let $K, L \subseteq N A$.

(1) If $K \subseteq L$ then $\mathrm{Rl} K \leqslant \mathrm{Rl} L$.

(2) $I \mathrm{Rl} K=\mathrm{Rl} I K$.

(3) $K \subseteq \mathrm{Rl} K$.

(4) $\mathrm{Rl} \mathrm{Rl} K \subseteq \mathrm{Rl} K$.

(5) $\mathrm{Rl} S K \subseteq S \mathrm{Rl} K$.

(6) $\mathrm{Rl}$ and $S \mathrm{Rl}$ are closure operators on classes of $N A$ 's.

Proof. (1) is obvious, and (2) is clear by general algebra. If $\mathscr{U} \in N A$ then $1 \in \operatorname{Sr} \mathfrak{A}$ by 1.13(9) and the IL, so (3) follows from 5.2(1). Let $\mathfrak{A} \in \mathrm{R} 1 \mathrm{R} 1 K$. Then $\mathfrak{U}=\mathfrak{R} \mathfrak{l}_{x} \mathfrak{R} \mathfrak{l}_{y} \mathfrak{B}$ for some $\mathfrak{B} \in K, y \in \operatorname{Sr} \mathfrak{B}$, and $x \in \operatorname{Sr} \mathfrak{R} \mathfrak{l}_{y} \mathfrak{B}$. Since $\mathfrak{A}=\mathfrak{R} \mathfrak{l}_{x} \mathfrak{B}$ by 5.2(2), we only need $x \in \operatorname{Sr} \mathfrak{B}$. From $x \in \operatorname{Sr} \mathfrak{A} \mathfrak{l}_{y} \mathfrak{B}$ we get $x=x^{\cup y}=x^{\cup} \cdot y=$ $x^{\cup} \cdot y^{\cup}=(x \cdot y)^{\cup}=x^{\cup}$ since $x \leqslant y$, and $x=\left(1{ }^{\prime} \cdot x\right) ;{ }^{y} x=(1 \cdot y \cdot x) ; x \cdot y \leqslant$ $(1 \cdot x) ; x \leqslant 1 ' ; x=x$, so $x \in \operatorname{Sr} \mathfrak{B}$. Thus (4) holds. (5) follows from 5.2(3) and the fact that $\operatorname{Sr} \mathfrak{A} \subseteq \operatorname{Sr} \mathfrak{B}$ whenever $\mathfrak{A} \subseteq \mathfrak{B}$. Finally, (6) follows from (1), (3)-(5).

THEOREM 5.8. (1) $\mathrm{Rl} N A=N A$.

(2) $\mathrm{Rl} W A=W A$.

Proof. Part (1) follows from 5.5 and 5.7(3). We shall prove $\mathrm{Rl} W A \subseteq W A$, from which (2) follows by 5.7(3).

Let $\mathfrak{A} \in W A$ and $x \in \operatorname{Sr} \mathfrak{A}$. Since $\mathfrak{R} \mathfrak{l}_{x} \mathfrak{A} \in N A$ by 5.5 , it suffices to show that $\mathfrak{R} \mathfrak{l}_{x} \mathfrak{A}$ satisfies the WL. Let $y \in \mathrm{Rl}_{x} \mathfrak{A}$. Then $\left(1_{x} \cdot y\right) ;{ }^{x} x \leqslant\left(1_{x} \cdot y\right) ;{ }^{x} x ;{ }^{x} x$ by $1.13(4)$, and

$$
\begin{array}{rlrl}
\left(1_{x}, y\right) ;{ }^{x} x ;{ }^{x} x & =\left(\left(1^{\prime} \cdot x \cdot y\right) ; x \cdot x\right) ; x \cdot x & \\
& \leqslant\left(1^{\prime} \cdot y\right) ; 1 ; 1 \cdot x & & 1.13(3) \\
& =\left(1^{\prime} \cdot y\right) ; 1 \cdot x & & \mathrm{WL} \\
& \leqslant\left(1^{\prime} \cdot y\right) ;\left(1 \cdot\left(1^{\prime} \cdot y\right)^{\cup} ; x\right) \cdot x & & 1.4(1) \\
& \leqslant\left(1^{\prime} \cdot y\right) ; x \cdot x & & 1.13(3),(12),(14), \mathrm{IL} \\
& =\left(1^{\prime} \cdot y\right) ;{ }^{x} x . &
\end{array}
$$

The next theorem shows that $S A, R A$, and $R R A$ are not closed under relativization.

THEOREM 5.9. Let $U$ be a set, and $R \in \mathrm{Sr} \Re$ e $U$.

(1) $R$ is transitive iff $\Re \mathfrak{l}_{R} \Re$ e $U=\subseteq \mathfrak{b} R$.

(2) $R$ is not transitive iff $\Re \mathfrak{l}_{R} \Re$ e $U \in W A \sim S A$.

Proof. $R$ is symmetric and reflexive over its field, since $R \in \operatorname{Sr} \Re$ e $U$. So if $R$ is transitive, then $R$ is an equivalence relation and $\Re \mathfrak{l}_{R} \Re$ e $U=\subseteq \mathfrak{b} R$ by 5.3(1).

Suppose $R$ is not transitive. We know that $\Re \mathfrak{I}_{R} \Re$ e $U \in W A$ by 5.8(2). Obviously $\mathfrak{R l} \mathfrak{l}_{R} \Re$ e $U$ is complete and atomic, so $\Re \mathfrak{l}_{R} \mathfrak{R}$ e $U \cong \mathbb{E} \mathrm{m} \mathfrak{A} \mathfrak{t} \mathfrak{R} \mathfrak{l}_{R} \mathfrak{R}$ e $U$ by 3.13(2). We shall show that $\mathfrak{A} \mathfrak{t} \mathfrak{R} \mathfrak{l}_{R} \mathfrak{R}$ e $U$ fails to satisfy $2.2(\mathrm{e})$, and hence $\mathfrak{R} \mathfrak{l}_{R} \Re$ e $U \notin S A$. Note that At $\Re \mathfrak{l}_{R} \Re$ e $U=\{\{\langle a, b\rangle\}:\langle a, b\rangle \in R\}$. Since $R$ is not transitive, there are $a, b$, 
$c \in U$ such that $\langle a, b\rangle,\langle b, c\rangle \in R$ but $\langle a, c\rangle \notin R$. Set $v=\{\langle b, a\rangle\}, w=\{\langle a, b\rangle\}$, $x=\{\langle b, b\rangle\}$, and $y=\{\langle b, c\rangle\}$. Then $w, y \in$ At $\mathfrak{R} \mathfrak{l}_{R} \Re$ e $U$, and $v, \quad x \in$ At $\Re \mathfrak{l}_{R} \mathfrak{R}$ e $U$ since $R$ is symmetric and reflexive. Then $\langle v, w, x\rangle,\langle x, y, y\rangle \in$ $C^{\left(\Re I_{R} \Re \mathrm{e} U\right)}$ but there is no $u \in$ At $\Re \mathfrak{l}_{R} \Re$ e $U$ such that $\langle v, u, y\rangle \in C^{\left(\Re \mathrm{I}_{R} \Re \mathrm{e} U\right)}$.

We have seen that $N A$ and $W A$ are closed under $\mathrm{Rl}$ and $\mathrm{SRl}$, while $S A, R A$, and $R R A$ are not; in fact, $\mathrm{Rl} R R A$ and $\mathrm{SRl} R R A$ contain complete atomic $W A$ 's which are not $S A$ 's. The rest of this section is devoted to the problem of characterizing Rl $R R A$ and SRl RRA. We will show that every complete atomic $W A$ is in $\mathrm{Rl} R R A$, and hence $W A=\mathrm{SRl} R R A$. The proof is divided into two parts. After a definition and two lemmas we will show in 5.13 that every complete atomic $W A$ is the relativization of a complete atomic $W A$ which satisfies a special condition. Then, after another definition and four more lemmas, we will show in 5.19 that any atomic $W A$ satisfying the special condition is in $R R A$.

Definition 5.10. Let $\mathfrak{A} \in N A$. Then $x^{d}=x ; x^{\cup} \cdot 1^{\prime}$ and $x^{r}=x^{\cup} ; x \cdot 1$ ' for every $x \in A$.

LEMMa 5.11. Let $\mathfrak{A} \in N A$. Then for every $x \in A$ :

(1) $\left(x^{\cup}\right)^{d}=x^{r}$ and $\left(x^{\cup}\right)^{r}=x^{d}$,

(2) if $x \leqslant 1$, then $x=x^{d}=x^{r}$,

(3) $x=x^{d} ; x=x ; x^{r}$.

Proof. (1) follows immediately from 1.13(8). Suppose $x \leqslant 1$ '. Then, using 1.13(3), (12), (14), 1.4(1), and the IL, we get $x^{d}=x ; x^{\cup} \cdot 1^{\prime} \leqslant x ; 1^{\prime} \cup 1^{\prime} \leqslant x=x ; 1^{\prime} \cdot 1^{\prime} \leqslant$ $x ;\left(1^{\prime} \cdot x^{\cup} ; 1^{\prime}\right) \cdot 1^{\prime} \leqslant x ; x^{\cup} \cdot 1^{\prime}=x^{d}$, so $x^{d}=x$. Now $x=x^{\cup}$ by $1.13(16)$, so $x^{r}=$ $\left(x^{\cup}\right)^{d}=x^{d}$ by part (1). Thus (2) holds. For (3), we use 1.4(2), 1.13(3), and the IL to get $x^{d} ; x \leqslant 1^{\prime} ; x=x=1 ' ; x \cdot x \leqslant\left(1^{\prime} \cdot x ; x^{\cup}\right) ; x=x^{d} ; x$. The proof of $x=x ; x^{r}$ is similar.

Lemma 5.12. Let $\mathfrak{A} \in W A$. Then for all $x, y, z \in$ At $\mathfrak{A}$ and $w \in I^{(\mathfrak{A})}$,

(1) $x^{d}, x^{r} \in$ At $\mathfrak{A}$,

(2) $w=x^{d}$ iff $x \leqslant w ; x$ iff $\langle w, x, x\rangle \in C^{(\mathfrak{A})}$,

(3) $w=x^{r}$ iff $x \leqslant x ; w$ iff $\langle x, w, x\rangle \in C^{(\mathfrak{A})}$,

(4) if $x \leqslant y$; then $x^{d}=y^{d}, y^{r}=z^{d}$, and $x^{r}=z^{r}$.

Proof. Part (1) is a restatement of 3.5. If $w=x^{d}$, then $x \leqslant w ; x$ by 5.11(3). Suppose $x \leqslant w ; x$. Then $0 \neq x=w ; x \cdot x \leqslant\left(w \cdot x ; x^{\cup}\right) ; x$ by 1.4(2). Since $w=w \cdot 1$ ' and ; is normal, we get $0 \neq w \cdot x ; x^{\cup}=w \cdot x^{d}$. Hence $w=x^{d}$ by part (1). We have shown $w=x^{d}$ iff $x \leqslant w ; x$. This completes the proof of (2), since $x \leqslant w ; x$ and $\langle w, x$, $x\rangle \in C^{(\mathfrak{A})}$ are equivalent by the definition of $C^{(\mathfrak{A})}$. The proof of (3) is similar.

Assume $x \leqslant y ; z$. Then

$$
\begin{aligned}
& x=y ; z \cdot x \\
& =y^{d} ; y ; z \cdot x \quad 5.11(3) \\
& \leqslant y^{d} ; 1 ; 1 \cdot x \quad 1.13(3) \\
& =y^{d} ; 1 \cdot x \quad \text { WL } \\
& \leqslant y^{d} ;\left(1 \cdot\left(y^{d}\right)^{\cup} ; x\right) \quad 1.4(1) \\
& \leqslant y^{d} ; x \quad 1.13(3),(12),(14), \mathrm{IL} \text {, }
\end{aligned}
$$


so $x^{d}=y^{d}$ by (1) and (2). From $0 \neq x=y ; z \cdot x$ we get $y^{\cup} ; x \cdot z \neq 0$ by the LPL, and $0 \neq z^{\cup} ; y^{\cup} \cdot x^{\cup}$ by 1.13(7), (8), (11), (13). Hence $z \leqslant y^{\cup} ; x$ and $x^{\cup} \leqslant z^{\cup} ; y^{\cup}$ since $x^{\cup} \in$ At $\mathfrak{A}$ by 3.4. By the argument above and 5.11(1), we get $z^{d}=\left(y^{\cup}\right)^{d}=y^{r}$ and $x^{r}=\left(x^{\cup}\right)^{d}=\left(z^{\cup}\right)^{d}=z^{r}$.

Theorem 5.13. Assume $\mathfrak{A} \in W A$ and $\mathfrak{A}$ is complete and atomic. Then $\mathfrak{A} \cong \mathfrak{R} \mathfrak{l}_{a} \mathfrak{B}$ for some $\mathfrak{B} \in W A$ and some $a \in \mathrm{Sr} \mathfrak{B}$, where $\mathfrak{B}$ is complete, atomic, and satisfies the following condition:

(*) for all $u, v \in I^{(\mathfrak{B})}$ there is some $x \in \mathrm{At} \mathfrak{B} \sim I^{(\mathfrak{B})}$ such that $x^{d}=u, x^{r}=v$, and $x \leqslant y$; $z$ whenever $y, z \in$ At $\mathfrak{B} \sim I^{(\mathfrak{B})}, y^{d}=u, y^{r}=z^{d}$, and $z^{r}=v$.

Proof. We shall assume, without loss of generality, that At $\mathfrak{A}$ and $I^{(\mathfrak{A})} \times I^{(\mathfrak{A})}$ are disjoint. Set $U=$ At $\mathfrak{A} \cup\left(I^{(\mathfrak{A})} \times I^{(\mathfrak{A})}\right)$. Define $f \in{ }^{U} U$ as follows:

$$
f x= \begin{cases}x^{\cup} & \text { if } x \in \text { At } \mathfrak{A} \\ \langle v, u\rangle & \text { if } x=\langle u, v\rangle, u, v \in I^{(\mathfrak{A})}\end{cases}
$$

Let $D$ be the subset of ${ }^{3} U$ consisting of all triples of the following types:

$$
\left\langle x, y,\left\langle x^{d}, y^{r}\right\rangle\right\rangle
$$$$
x^{r}=y^{d}, x, y \in \text { At } \mathfrak{A} \sim I^{(\mathfrak{A})},
$$$$
\text { (Ib) }\left\langle x,\left\langle x^{r}, y^{r}\right\rangle, y\right\rangle
$$$$
x^{d}=y^{d}, x, y \in \text { At } \mathfrak{A} \sim I^{(\mathfrak{A})},
$$$$
\text { (Ic) }\left\langle\left\langle y^{d}, x^{d}\right\rangle, x, y\right\rangle
$$$$
x^{r}=y^{r}, x, y \in \text { At } \mathfrak{A} \sim I^{(\mathfrak{A})} \text {, }
$$

(IIa) $\left\langle\left\langle x^{d}, u\right\rangle,\left\langle u, x^{r}\right\rangle, x\right\rangle$ $u \in I^{(\mathfrak{A})}, x \in$ At $\mathfrak{U}$,

(IIb) $\left\langle x,\left\langle x^{r}, u\right\rangle,\left\langle x^{d}, u\right\rangle\right\rangle$ $u \in I^{(\mathfrak{A})}, x \in$ At $\mathfrak{U}$,

(IIc) $\left\langle\left\langle u, x^{d}\right\rangle, x,\left\langle u, x^{r}\right\rangle\right\rangle$ $u \in I^{(\mathfrak{A})}, x \in$ At $\mathfrak{A}$,$$
\langle\langle u, v\rangle,\langle v, w\rangle,\langle u, w\rangle\rangle
$$

$u, v, w \in I^{(\mathfrak{A})}$.

Let $C^{\prime}=C^{(\mathfrak{A})} \cup D$. Finally, let $\mathfrak{U}=\left\langle U, C^{\prime}, f, I^{(\mathfrak{A})}\right\rangle$ and $\mathfrak{B}=\mathfrak{S} \mathfrak{m} \mathfrak{U}$. Notice that $C^{\prime} \cap^{3}$ At $\mathfrak{A}=C^{(\mathfrak{A})}$ and the restriction of $f$ to At $\mathfrak{U}$ is ${ }^{U}$. Consequently $\mathfrak{A} \mathfrak{t} \mathfrak{A}$ is a substructure of $\mathfrak{H}$.

We shall prove that $\mathfrak{E} \mathfrak{m} \mathfrak{U} \in W A$ by verifying $2.2(\mathrm{a})-(\mathrm{d})$. For $2.2(\mathrm{a})$, (b), suppose $\langle x, y, z\rangle \in C^{\prime}$. If $\langle x, y, z\rangle \in C^{(\mathfrak{A})}$, then $\langle f x, z, y\rangle=\left\langle x^{\cup}, z, y\right\rangle \in C^{(\mathfrak{A})} \subseteq C^{\prime}$ and $\langle z, f y, x\rangle=\left\langle z, y^{\cup}, x\right\rangle \in C^{(\mathfrak{A})} \subseteq C^{\prime}$ since $\mathfrak{A} \mathrm{t} \mathfrak{A}$ satisfies 2.2(a), (b). If $\langle x, y, z\rangle$ is a triple of type (Ia), then $\langle x, y, z\rangle=\left\langle x, y,\left\langle x^{d}, y^{r}\right\rangle\right\rangle$ where $x^{r}=y^{d}$ and $x, y \in$ At $\mathfrak{A}$ $\sim I^{(\mathfrak{A})}$. But then $\left(x^{\cup}\right)^{d}=y^{d}$ by 5.11(1) and $x^{\cup}, y \in$ At $\mathfrak{A} \sim I^{(\mathfrak{A})}$ by 3.4 and 1.13(16), so $\left\langle f x,\left\langle x^{d}, y^{r}\right\rangle, y\right\rangle=\left\langle x^{\cup},\left\langle\left(x^{\cup}\right)^{r}, y^{r}\right\rangle, y\right\rangle$ is a triple of type (Ib). Similar observations show that every transform of a triple of type (I) is also type (I). It is even easier to see that if $\langle x, y, z\rangle$ is type (II) or (III), then so are all of its transforms (nothing more than the definition of $f$ is needed). Thus $\mathfrak{H}$ satisfies 2.2(a), (b).

Next we verify $2.2(\mathrm{c})$ in one direction. Let $x \in U$. If $x \in$ At $\mathfrak{A}$, then $\langle x, w, x\rangle \in$ $C^{(\mathfrak{A})} \subseteq C^{\prime}$ for some $w \in I^{(\mathfrak{A})}$ since $\mathfrak{A} t \mathfrak{A}$ satisfies 2.2(c). Suppose $x=\langle u, v\rangle$ where $u, v \in I^{(\mathscr{H})}$. Then $v=v^{d}=v^{r}$ by $5.11(2)$, so $\langle x, v, x\rangle=\langle\langle u, v\rangle, v,\langle u, v\rangle\rangle$ is a triple of type (IIc). Now, for the other direction, suppose $\langle x, w, y\rangle \in C^{\prime}$ with $w \in I^{(\mathfrak{A})}$. If $x, y \in$ At $\mathfrak{A}$, then $\langle x, w, y\rangle \in C^{(\mathfrak{A})}$ and $x=y$ since $\mathfrak{A} \mathfrak{t} \mathfrak{A}$ satisfies 2.2(c). Suppose $x=\langle u, v\rangle$ where $u, v \in I^{(\mathfrak{A})}$. Then $\langle x, w, y\rangle$ must be type (IIc). 
(Type (Ic) is ruled out since $w \notin$ At $\mathfrak{A} \sim I^{(\mathfrak{A})}$.) Consequently $x=\left\langle u, w^{d}\right\rangle$ and $y=\left\langle u, w^{r}\right\rangle$. But $w=w^{d}=w^{r}$ by 5.11(2), so $x=y$. This completes the proof of 2.2(c) for $\mathfrak{U}$.

For 2.2(d) we must show $\langle w, z, z\rangle \in C^{\prime}$ whenever $w \in I^{(\mathfrak{R})}$ and $\langle w, x, x\rangle$, $\langle x, y, z\rangle \in C^{\prime}$. This certainly holds when $x, y, z \in$ At $\mathfrak{A}$ since $\mathfrak{A} t \mathfrak{A}$ satisfies 2.2(d). There are seven other cases depending on the type of $\langle x, y, z\rangle$. These cases are listed below. Throughout the list we assume $q, u, v, w \in I^{(\mathfrak{A})}$ and $x, y \in$ At $\mathfrak{A}$.

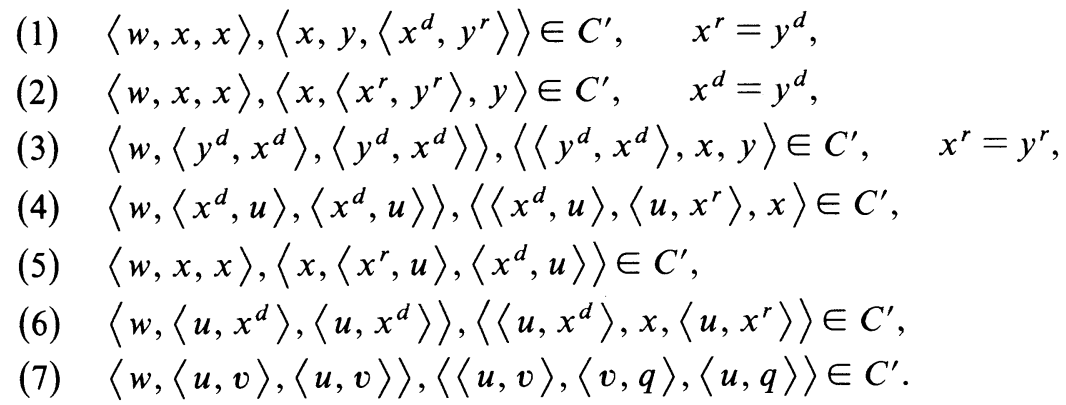

Case (1). We get $w=x^{d}$ by $5.12(2)$, so $\left\langle w,\left\langle x^{d}, y^{r}\right\rangle,\left\langle x^{d}, y^{r}\right\rangle\right\rangle$ is a triple of type (IIb).

Case (2). By 5.12(2), $w=x^{d}$. But $x^{d}=y^{d}$, so $\langle w, y, y\rangle \in C^{(\mathfrak{A})}$ by 5.12(2).

Case (3). The first triple can only be type (IIb), so $y^{d}=w^{d}$. Then $y^{d}=w$ by 5.11(2), and $\langle w, y, y\rangle \in C^{(\mathfrak{I})}$ by 5.12(2).

Case (4). Similar to case (3).

Case (5). Similar to case (1).

Case (6). The first triple must be type (IIb), so $u=w^{d}=w$ by 5.11(2). Then $\left\langle w,\left\langle u, x^{r}\right\rangle,\left\langle u, x^{r}\right\rangle\right\rangle$ is type (IIb) as well.

Case (7). Similar to case (6).

This completes the proof of $\mathfrak{C} \mathfrak{m} \mathfrak{U} \in W A$.

Consider At $\mathfrak{A}$ as an element of $\mathfrak{C} \mathfrak{m} \mathfrak{U}$. We have (At $\mathfrak{U})^{\cup}=\{f x: x \in$ At $\mathfrak{A}\}=$ $\left\{x^{\cup}: x \in\right.$ At $\left.\mathfrak{A}\right\}=$ At $\mathfrak{A}$ by 3.4 and $1.13(8)$, and $\left(I^{(\mathfrak{A})} \cap\right.$ At $\left.\mathfrak{A}\right) ;$ At $\mathfrak{U}=I^{(\mathfrak{A})} ;$ At $\mathfrak{A}$ $=$ At $\mathfrak{A}$ by the IL for $\mathfrak{C} \mathfrak{m} \mathfrak{U}$, so At $\mathfrak{A} \in \operatorname{Sr} \mathfrak{C} \mathfrak{m} \mathfrak{U}$. It is easy to check that $\mathfrak{R} \mathfrak{I}_{\text {At } \mathfrak{Y}} \mathfrak{E} \mathfrak{m} \mathfrak{U}=\mathfrak{E} \mathfrak{m} \mathfrak{A} t \mathfrak{A}$. The crucial fact, which is needed to show that these two algebras have the same relative product, is that $C^{\prime} \cap^{3}$ At $\mathscr{A}=C^{(\mathfrak{A})}$. We have $\mathfrak{U} \cong \mathfrak{C} \mathfrak{m} \mathfrak{A} \mathfrak{t} \mathfrak{A}$ by 3.13 , so, setting $a=$ At $\mathfrak{A}$ and $\mathfrak{B}=\mathfrak{C} \mathfrak{m} \mathfrak{U}$, we get $\mathfrak{B} \in W A$, $a \in \operatorname{Sr} \mathfrak{B}, \mathfrak{A} \cong \mathfrak{C} \mathfrak{m} \mathfrak{A} t \mathfrak{A}=\mathfrak{R} \mathfrak{l}_{\text {At } \mathfrak{U}} \mathfrak{E} \mathfrak{m} \mathfrak{U}=\mathfrak{R} \mathfrak{l}_{a} \mathfrak{B}$, and $\mathfrak{B}$ is complete and atomic. It remains to show that $\mathscr{B}$ satisfies $(*)$.

First notice that At $\mathfrak{B}=\{\{x\}: x \in$ At $\mathfrak{A}\} \cup\left\{\{\langle u, v\rangle\}: u, v \in I^{(\mathfrak{Q})}\right\}$. If $x \in$ At $\mathfrak{A}$ then $\{x\}^{d}=I^{(\mathscr{Q})} \cap\{x\} ;\{x\}^{\cup}=\left\{u: u \in I^{(\mathfrak{Q})}\right.$ and $\left.\left\langle x, x^{\cup}, u\right\rangle \in C^{\prime}\right\}=\left\{u: u \in I^{(\mathscr{U})}\right.$ and $\left.\langle u, x, x\rangle \in C^{(\mathfrak{I})}\right\}$ since $C^{\prime} \cap^{3}$ At $\mathfrak{U}=C^{(\mathfrak{A})}$. Consequently $\{x\}^{d}=\left\{x^{d}\right\}$ by 5.12(2). Similarly, $\{x\}^{r}=\left\{x^{r}\right\}$. If $u, v \in I^{(\mathfrak{U})}$, then $\{\langle u, v\rangle\}^{d}=I^{(\mathfrak{U})} \cap\{\langle u, v\rangle\}$; $\{\langle u, v\rangle\}^{\cup}=\left\{w: w \in I^{(\mathscr{Q})}\right.$ and $\left.\langle w,\langle u, v\rangle,\langle u, v\rangle\rangle \in C^{\prime}\right\}$. So $w \in\{\langle u, v\rangle\}^{d}$ iff $w \in$ $I^{(\mathfrak{Q})}$ and $\langle w,\langle u, v\rangle,\langle u, v\rangle\rangle$ is a triple of type (IIb), which occurs iff $u=w^{d}=w$ by 5.11(2). Thus $\{\langle u, v\rangle\}^{d}=\{u\}$, and similarly $\{\langle u, v\rangle\}^{r}=\{v\}$. 
Let $u^{\prime}, v^{\prime} \in I^{(\mathfrak{B})}$. Then $u^{\prime}=\{u\}$ and $v^{\prime}=\{v\}$ for some $u, v \in I^{(\mathfrak{A})}$. Set $x^{\prime}=$ $\{\langle u, v\rangle\}$. Note that $x^{\prime} \in$ At $\mathfrak{B} \sim I^{(\mathfrak{B})}$. Let $y^{\prime}, z^{\prime} \in$ At $\mathfrak{B} \sim I^{(\mathfrak{B})}$, and assume

$$
\left(y^{\prime}\right)^{d}=\{u\}, \quad\left(y^{\prime}\right)^{r}=\left(z^{\prime}\right)^{d}, \quad \text { and } \quad\left(z^{\prime}\right)^{r}=\{v\} .
$$

We must show $x^{\prime}=\{\langle u, v\rangle\} \subseteq y^{\prime} ; z^{\prime}$. The proof splits into four cases.

Case (a). $y^{\prime}=\{y\}, z^{\prime}=\{z\}, y, z \in$ At $\mathfrak{A} \sim I^{(\mathfrak{A})}$. From $(* *)$ we get $y^{d}=u$, $y^{r}=z^{d}$, and $z^{r}=v$. Then $\langle y, z,\langle u, v\rangle\rangle=\left\langle y, z,\left\langle y^{d}, z^{r}\right\rangle\right\rangle$ is a triple of type (Ia), so $x^{\prime} \subseteq y^{\prime} ; z^{\prime}$.

Case (b). $y^{\prime}=\{y\}, y \in$ At $\mathfrak{A} \sim I^{(\mathfrak{A})}, z^{\prime}=\{\langle q, w\rangle\}, q, w \in I^{(\mathfrak{A})}$. Using (**) we get $y^{d}=u, y^{r}=q$, and $w=v$. Then $\langle y,\langle q, w\rangle,\langle u, v\rangle\rangle=\left\langle y,\left\langle y^{r}, v\right\rangle,\left\langle y^{d}, v\right\rangle\right\rangle$ is a triple of type (IIb) and $x^{\prime} \subseteq y^{\prime} ; z^{\prime}$.

Case (c). $y^{\prime}=\{\langle q, w\rangle\}, q, w \in I^{(\mathfrak{A})}, z^{\prime}=\{z\}, z \in$ At $\mathfrak{A} \sim I^{(\mathfrak{A})}$. From $(* *)$ we have $q=u, w=z^{d}$, and $z^{r}=v$. Then $\langle\langle q, w\rangle, z,\langle u, v\rangle\rangle=\left\langle\left\langle u, z^{d}\right\rangle, z,\left\langle u, z^{r}\right\rangle\right\rangle$ is a triple of type (IIc), so $x^{\prime} \subseteq y^{\prime} ; z^{\prime}$.

Case (d). $y^{\prime}=\{\langle q, w\rangle\}, z^{\prime}=\{\langle s, t\rangle\}, q, w, s, t \in I^{(\mathfrak{A})}$. By $(* *), q=u, w=s$, and $t=v$, so $\langle\langle q, w\rangle,\langle s, t\rangle,\langle u, v\rangle\rangle=\langle\langle u, w\rangle,\langle w, v\rangle,\langle u, v\rangle\rangle$ is a triple of type (III), and hence $x^{\prime} \subseteq y^{\prime} ; z^{\prime}$.

Definition 5.14. Assume $\mathfrak{A} \in N A$ and $\alpha$ is an ordinal. Then $L_{\alpha} \mathfrak{A}$ is the set of all $l \in{ }^{\alpha \times \alpha}$ At $\mathfrak{A}$ satisfying the following conditions for all $\kappa, \lambda, \mu<\alpha$.

(1) $l_{k \lambda} \leqslant 1$ ' iff $\kappa=\lambda$,

(2) $l_{\kappa \lambda}^{\cup}=l_{\lambda \kappa}$,

(3) $l_{\kappa \lambda} \leqslant l_{\kappa \mu} ; l_{\mu \lambda}$.

The elements of $L_{\alpha} \mathfrak{A}$ are called $\mathfrak{A}$-labellings of $\alpha$. Let $l \in L_{\alpha} \mathfrak{A}$. A flaw in $l$ is a quadruple $\langle\kappa, \lambda, x, y\rangle$ where $\kappa, \lambda<\alpha, x, y \in$ At $\mathfrak{A}, l_{\kappa \lambda} \leqslant x ; y$, and for every $\mu<\alpha$, either $l_{\kappa \mu} \neq x$ or $l_{\mu \lambda} \neq y$. The labelling $l$ is complete if there are no flaws in $l$.

Lemma 5.15. Assume $\mathfrak{A} \in W A, \mathfrak{A}$ is atomic, $l$ is a complete $\mathfrak{A}$-labelling of $\alpha$, and $l$ maps $\alpha \times \alpha$ onto At $\mathfrak{A}$. Then $\mathfrak{A}$ is isomorphic to a subalgebra of $\Re$ e $\alpha$.

Proof. Define $R(x)=\left\{\langle\kappa, \lambda\rangle: \kappa, \lambda<\alpha\right.$ and $\left.l_{\kappa \lambda} \leqslant x\right\}$ for all $x \in A$. Clearly, if $x \leqslant y$ then $R(x) \subseteq R(y)$. Suppose $x \neq y$. Then $x \cdot-y \neq 0$, so $a \leqslant x \cdot-y$ for some $a \in$ At $\mathfrak{U}$ since $\mathfrak{U}$ is atomic. Since $l$ is onto, there are $\kappa, \lambda<\alpha$ such that $l_{\kappa \lambda}=a \leqslant x$ - $-y$. But then $\langle\kappa, \lambda\rangle \in R(x)$ and $\langle\kappa, \lambda\rangle \notin R(y)$, so $R(x) \nsubseteq R(y)$. We have shown

$$
x \leqslant y \quad \text { iff } \quad R(x) \subseteq R(y), \quad \text { for all } x, y \in A .
$$

Clearly $R(0)=\varnothing$ and $R(1)=\alpha \times \alpha$. Consequently $R$ is an embedding of $\mathfrak{B} \mathfrak{l} \mathfrak{A}$ into $\mathfrak{B l} \mathfrak{R}$ e $\alpha$.

We get $\mathrm{Id}_{\alpha}=R\left(1^{\prime}\right)$ from $5.14(1)$, and $R\left(x^{\cup}\right)=R(x)^{-1}$ from 5.14(2). It follows from 5.14(3) that $R(x) \mid R(y) \subseteq R(x ; y)$, while $R(x ; y) \subseteq R(x) \mid R(y)$ since $l$ is complete. Thus $R$ is an embedding of $\mathfrak{A}$ into $\Re$ e $\alpha$.

LEMma 5.16. If $\mathfrak{A} \in W A, \mathfrak{A}$ satisfies 5.13(*), $l \in L_{\alpha} \mathfrak{A}$, and $\langle\kappa, \lambda, x, y\rangle$ is a flaw in $l$, then there is some $m \in L_{\alpha+1} \mathfrak{A}$ such that $l \subseteq m, m_{\kappa \alpha}=x$, and $m_{\alpha \lambda}=y$ (i.e. $\langle\kappa, \lambda, x, y\rangle$ is not a flaw in $m$ ). 
Proof. Since $\mathfrak{A}$ satisfies 5.13(*), there is a function $a$, mapping $I^{(\mathfrak{A})} \times I^{(\mathfrak{A})}$ into At $\mathfrak{A} \sim I^{(\mathfrak{A})}$, such that for all $u, v \in I^{(\mathfrak{A})}$ we have

(1) $a(u, v)^{d}=u$,

(2) $a(u, v)^{r}=v$,

(3) if $w, z \in$ At $\mathfrak{A}, w^{d}=u, w^{r}=z^{d}$, and $z^{r}=v$, then $a(u, v) \leqslant w ; z$.

Using 3.4 and 5.12(1), we define $m \in \in^{(\alpha+1) \times(\alpha+1)}$ At $\mathfrak{A}$ as follows:

$$
\begin{aligned}
& m_{\mu \nu}=l_{\mu \nu} \quad \text { for all } \mu, \nu<\alpha, \\
& m_{\kappa \alpha}=x, \quad m_{\alpha \kappa}=x^{\cup}, \quad m_{\alpha \lambda}=y, \\
& m_{\lambda \alpha}=y^{\cup}, \quad m_{\alpha \alpha}=x^{r}, \quad m_{\mu \alpha}=a\left(l_{\mu \mu}, x^{r}\right), \\
& m_{\alpha \mu}=a\left(l_{\mu \mu}, x^{r}\right)^{\cup} \quad \text { if } \mu<\alpha \text { and } \mu \neq \kappa, \lambda .
\end{aligned}
$$

We have apparently assumed $\kappa \neq \lambda$ in the definition of $m$. However, suppose $\kappa=\lambda$. Then $0 \neq l_{\kappa \kappa}=l_{\kappa \lambda} \leqslant x ; y \cdot 1^{\prime}$ by $5.14(1)$, so $0 \neq x^{\cup} ; 1^{\prime} \cdot y=x^{\cup} \cdot y$ by the LPL, and $0 \neq x \cdot y^{\cup}$ by the RPL. Thus $y=x^{\cup}$ and $y^{\cup}=x$, so the definition of $m$ is merely redundant.

The proof will be complete as soon as we show $m \in L_{\alpha+1} \mathfrak{A}$. To verify 5.14(2) for $m$ it suffices, since $m \supseteq l \in L_{\alpha} \mathfrak{A}$, to show $l_{\alpha \alpha}^{\cup}=l_{\alpha \alpha}, l_{\mu \alpha}^{\cup}=l_{\alpha \mu}$, and $l_{\alpha \mu}^{\cup}=l_{\mu \alpha}$ for all $\mu<\alpha$. These formulas follow from 1.13(8), (16). Obviously $m$ satisfies half of $5.14(1)$, namely $m_{\mu \mu} \leqslant 1$ ' for all $\mu<\alpha+1$. We will prove the other half of $5.14(1)$ later.

Before continuing we make some observations which will simplify the proof that $l$ satisfies 5.14(3).

$$
x^{d}=m_{\kappa \kappa}, \quad x^{r}=y^{d}, \quad \text { and } y^{r}=m_{\lambda \lambda} .
$$

Proof. Since $\langle\kappa, \lambda, x, y\rangle$ is a flaw in $l$, we have $l_{\kappa \lambda} \leqslant x ; y$, and so, by $5.12(4)$, $l_{\kappa \lambda}^{d}=x^{d}, x^{r}=y^{d}$, and $l_{\kappa \lambda}^{r}=y^{r}$. Since $l \in L_{\alpha} \mathfrak{A}$, we have $l_{\kappa \kappa}, l_{\lambda \lambda} \leqslant 1, l_{\kappa \lambda} \leqslant l_{\kappa \kappa} ; l_{\kappa \lambda}$, and $l_{\kappa \lambda} \leqslant l_{\kappa \lambda} ; l_{\lambda \lambda}$. Hence $l_{\kappa \lambda}^{d}=l_{\kappa \kappa}=m_{\kappa \kappa}$ and $l_{\kappa \lambda}^{r}=l_{\lambda \lambda}=m_{\lambda \lambda}$ by 5.12(2), (3).

Thus $x^{d}=l_{\kappa \lambda}^{d}=m_{\kappa \kappa}$ and $y^{r}=l_{\kappa \lambda}^{r}=m_{\lambda \lambda}$.

$$
m_{\mu \nu}^{d}=m_{\mu \mu} \text { for all } \mu, \nu<\alpha+1 \text {. }
$$

Proof. Suppose $\mu, \nu<\alpha$. Then $m_{\mu \mu}=l_{\mu \mu} \leqslant 1$ ' and $m_{\mu \nu}=l_{\mu \nu} \leqslant l_{\mu \mu} ; l_{\mu \nu}=m_{\mu \mu} ; m_{\mu \nu}$, so $m_{\mu \nu}^{d}=m_{\mu \mu}$ by 5.12(2). Thus we may assume $\alpha \in\{\mu, \nu\}$. If $\alpha=\mu=\nu$, then $m_{\mu \nu}^{d}=m_{\alpha \alpha}^{d}=\left(x^{r}\right)^{d}=x^{r}=m_{\alpha \alpha}=m_{\mu \mu}$ by 5.11(2), so either $\mu<\alpha=\nu$ or $\nu<\alpha=\mu$.

Suppose $\mu<\alpha=\nu$. If $\mu \neq \kappa, \lambda$, then $m_{\mu \nu}^{d}=m_{\mu \alpha}^{d}=a\left(l_{\mu \mu}, x^{r}\right)^{d}=l_{\mu \mu}=m_{\mu \mu}$ by (1). If $\mu=\kappa$, then $m_{\mu \nu}^{d}=m_{\kappa \alpha}^{d}=x^{d}=m_{\kappa \kappa}=m_{\mu \mu}$ by (4). If $\mu=\lambda$, then $m_{\mu \nu}^{d}=m_{\lambda \alpha}^{d}=$ $\left(y^{\cup}\right)^{d}=y^{r}=m_{\lambda \lambda}=m_{\mu \mu}$ by (4).

Suppose $\nu<\alpha=\mu$. If $\nu \neq \kappa, \lambda$, then $m_{\mu \nu}^{d}=m_{\alpha \nu}^{d}=\left(a\left(l_{\nu \nu}, x^{r}\right)^{\cup}\right)^{d}=a\left(l_{\nu \nu}, x^{r}\right)^{r}=$ $x^{r}=m_{\alpha \alpha}=m_{\mu \mu}$ by (2). If $\nu=\kappa$ then $m_{\mu \nu}^{d}=m_{\alpha \kappa}^{d}=\left(x^{\cup}\right)^{d}=x^{r}=m_{\alpha \alpha}=m_{\mu \mu}$. If $\nu=\lambda$, then $m_{\mu \nu}^{d}=m_{\alpha \lambda}^{d}=y^{d}=x^{r}=m_{\alpha \alpha}=m_{\mu \mu}$ by (4).

$$
m_{\mu \nu}^{r}=m_{\nu \nu} \text { for all } \mu, \nu<\alpha+1 \text {. }
$$

Proof. Using (5) and 5.14(2) for $m$, we have $m_{\mu \nu}^{r}=\left(m_{\mu \nu}^{\cup}\right)^{d}=m_{\nu \mu}^{d}=m_{\nu \nu}$.

(7) For all $\mu, \nu, \xi<\alpha+1$, the six formulas which can be obtained from $m_{\mu \nu} \leqslant m_{\mu \xi} ; m_{\xi \nu}$ by permuting the subscripts $\mu, \nu, \xi$ are equivalent. 
Proof. This follows from the Peircean laws, $m \in{ }^{(\alpha+1) \times(\alpha+1)}$ At $\mathfrak{A}$, and $5.14(2)$ for $m$. For example, if $m_{\mu \nu} \leqslant m_{\mu \xi} ; m_{\xi \nu}$, then $0 \neq m_{\mu \nu}=m_{\mu \xi} ; m_{\xi \nu} \cdot m_{\mu \nu}$, so $0 \neq$ $m_{\mu \xi}^{\cup} ; m_{\mu \nu} \cdot m_{\xi \mu}=m_{\xi \mu} ; m_{\mu \nu} \cdot m_{\xi \nu}$, and hence $m_{\xi \nu} \leqslant m_{\xi \mu} ; m_{\mu \nu}$.

Now we can show $m$ satisfies $5.14(3)$. Let $\mu, \nu, \xi<\alpha+1$. We wish to show $m_{\mu \nu} \leqslant m_{\mu \xi} ; m_{\xi \nu}$. Suppose $\mu, \nu, \xi$ are not distinct. We may assume $\mu=\xi$ by (7). Then by (5) and 5.11(3), $m_{\mu \nu}=m_{\mu \nu}^{d} ; m_{\mu \nu}=m_{\mu \mu} ; m_{\mu \nu}=m_{\mu \xi} ; m_{\xi \nu}$.

Suppose $\mu, \nu, \xi$ are distinct. If $\mu, \nu, \xi<\alpha$, then $m_{\mu \nu}=l_{\mu \nu} \leqslant l_{\mu \xi} ; l_{\xi \nu}=m_{\mu \xi} ; m_{\xi \nu}$ since $l \in L_{\alpha} \mathfrak{A}$, so we may assume, by (7), that $\nu=\alpha$. Thus $\mu, \xi<\alpha=\nu$. Suppose $\{\mu, \xi\} \neq\{\kappa, \lambda\}$. We may assume, by (7), that $\mu \neq \kappa, \lambda$. Then $m_{\mu \nu}=m_{\mu \alpha}=a\left(l_{\mu \mu}, x^{r}\right)$, and from (5) and (6) we get $l_{\mu \mu}=m_{\mu \mu}=m_{\mu \xi}^{d}, m_{\mu \xi}^{r}=m_{\xi \xi}=m_{\xi \nu}^{d}$, and $m_{\xi \alpha}^{r}=m_{\alpha \alpha}=$ $x^{r}$, so by (1)-(3), $m_{\mu \nu}=a\left(l_{\mu \mu}, x^{r}\right) \leqslant m_{\mu \xi} ; m_{\xi \nu}$. Suppose $\{\mu, \xi\}=\{\kappa, \lambda\}$. By (7) we may assume $\mu=\kappa$ and $\xi=\lambda$ since $\mu \neq \xi$. We have $l_{\kappa \lambda} \leqslant x ; y$ since $\langle\kappa, \lambda, x, y\rangle$ is a flaw in $l$, so $m_{\mu \xi}=m_{\kappa \lambda}=l_{\kappa \lambda} \leqslant x ; y=m_{\kappa \alpha} ; m_{\alpha \lambda}=m_{\mu \nu} ; m_{\nu \xi}$. Hence $m_{\mu \nu} \leqslant m_{\mu \xi} ; m_{\xi \nu}$ by (7). Thus $m$ satisfies 5.14(3).

Finally, we show that $\mu=\nu$ whenever $m_{\mu \nu} \leqslant 1$, so that $m$ satisfies 5.14(1). If $\mu$, $\nu<\alpha$ then $m_{\mu \nu}=l_{\mu \nu} \leqslant 1$, and $\mu=\nu$ since $l \in L_{\alpha} \mathfrak{A}$. Suppose $\mu<\alpha=\nu$ and $m_{\mu \nu} \leqslant 1$ '. Then $x=m_{\kappa \alpha} \leqslant m_{\kappa \mu} ; m_{\mu \alpha}=m_{\kappa \mu} ; m_{\mu \nu} \leqslant m_{\kappa \mu} ; 1^{\prime}=m_{\kappa \mu}$, so $x=m_{\kappa \mu}$. Also $y=m_{\alpha \lambda} \leqslant m_{\alpha \mu} ; m_{\mu \lambda}=m_{\nu \mu} ; m_{\mu \lambda}=m_{\mu \nu}^{\cup} ; m_{\mu \lambda} \leqslant 1$, $; m_{\mu \lambda}=m_{\mu \lambda}$, and thus $y=m_{\mu \lambda}$. But then $x=m_{\kappa \mu}=l_{\kappa \mu}$ and $y=m_{\mu \lambda}=l_{\mu \lambda}$, contradicting the hypothesis that $\langle\kappa, \lambda, x, y\rangle$ is a flaw in $l$.

LEMma 5.17. If $\mathfrak{A} \in W A, \mathfrak{A}$ satisfies 5.13(*), and $l \in L_{\alpha} \mathfrak{A}$, then there are some $\beta \geqslant \alpha$ and some $m \in L_{\beta} \mathfrak{A}$ such that $l \subseteq m$ and if $\langle\kappa, \lambda, x, y\rangle$ is a flaw in $m$, then $\alpha \leqslant \kappa, \lambda$.

Proof. If $l$ is complete, set $\beta=\alpha$ and $m=l$. Otherwise, let $\left\langle\left\langle\kappa_{\xi}, \lambda_{\xi}, x_{\xi}, y_{\xi}\right\rangle: \xi<\right.$ $\gamma\rangle$ be an enumeration of the flaws in $l$. Set $\beta=\alpha+\gamma$ and $m^{0}=l$. Suppose $\delta<\gamma$ and $m^{\delta}$ has been constructed. If $\left\langle\kappa_{\delta}, \lambda_{\delta}, x_{\delta}, y_{\delta}\right\rangle$ is a flaw in $m^{\delta}$, let $m^{\delta+1}$ be the $\mathfrak{A}$-labelling obtained by applying 5.16 to $m^{\delta}$. For any limit ordinal $\mu<\gamma$, let $m^{\mu}=\cup_{\delta<\mu} m^{\delta}$. Finally, let $m=\cup_{\delta<\gamma} m^{\delta}$. It is then easy to show that $l \subseteq m \in L_{\beta} \mathfrak{A}$ and $m$ has no flaws $\langle\kappa, \lambda, x, y\rangle$ with $\kappa, \lambda<\alpha$.

Lemma 5.18. If $\mathfrak{A} \in W A, \mathfrak{A}$ satisfies 5.13(*), and $l \in L_{\alpha} \mathfrak{A}$, then there is some complete $m \in L_{\beta}$ Ir such that $l \subseteq m$.

Proof. Set $m^{0}=l$, and for each $\kappa<\omega$ let $m^{\kappa+1}$ be the $\mathfrak{A}$-labelling obtained by applying 5.17 to the previously constructed labelling $m^{\kappa}$. Set $m=\cup_{\kappa<\omega} m^{\kappa}$. Then $m$ is a complete $\mathfrak{A}$-labelling which contains $l$.

Theorem 5.19. If $\mathfrak{A} \in W A, \mathfrak{A}$ is atomic, and $\mathfrak{A}$ satisfies 5.13(*), then $\mathfrak{A}$ is isomorphic to a subalgebra of $\Re$ e $\alpha$, for some ordinal $\alpha$.

Proof. Since $\mathfrak{A}$ satisfies 5.13(*), there is a function $a$, mapping $I^{(\mathfrak{A})} \times I^{(\mathfrak{A})}$ into At $\mathfrak{A} \sim I^{(\mathfrak{A})}$, which satisfies 5.16(1)-(3). Let $\gamma=\left|I^{(\mathfrak{A})}\right|$, and let $u$ be a one-to-one function mapping $\gamma$ onto $I^{(\mathfrak{A})}$. Define $l \in{ }^{\gamma \times \gamma}$ At $\mathfrak{A}$ as follows, for all $\kappa, \lambda<\gamma$ :

$$
l_{\kappa \lambda}= \begin{cases}u_{\kappa} & \text { if } \kappa=\lambda, \\ a\left(u_{\kappa}, u_{\lambda}\right) & \text { if } \kappa<\lambda, \\ a\left(u_{\kappa}, u_{\lambda}\right)^{\cup} & \text { if } \lambda<\kappa .\end{cases}
$$


Using 1.13(8), (16), 5.12(2), (3), and 5.16(1)-(3) one can easily prove that $l \in L_{\gamma} \mathfrak{A}$. By 5.18 there is some complete $m \in L_{\alpha} \mathfrak{U}$ such that $l \subseteq m$. To complete the proof it suffices, by 5.15 , to show $m$ is onto.

Let $x \in$ At $\mathfrak{A}$. If $x \in I^{(\mathfrak{A})}$ then $x=u_{\kappa}=l_{\kappa \kappa}=m_{\kappa \kappa}$ for some $\kappa<\gamma \leqslant \alpha$, so we may assume $x \notin I^{(\mathfrak{Y})}$. Choose $\kappa, \lambda<\gamma$ so that $u_{\kappa}=x^{d}$ and $u_{\lambda}=x^{r}$. Then $a\left(x^{d}, x^{r}\right)$ $=l_{\kappa \lambda}=m_{\kappa \lambda}$. Set $y=a\left(x^{r}, x^{r}\right)$. Note that $y \in$ At $\mathfrak{A} \sim I^{(\mathfrak{A})}, m_{\kappa \lambda}^{d}=x^{d}, x^{r}=y^{d}$, and $m_{\kappa \lambda}^{r}=y^{r}$ by 5.16(1), (2). Consequently $m_{\kappa \lambda} \leqslant x ; y$ by 5.16(3). Since $m$ is complete, there is some $\mu<\alpha$ such that $m_{\kappa \mu}=x$ and $m_{\mu \lambda}=y$. Thus $x$ is in the range of $m$.

THEOREM 5.20. (1) If $\mathfrak{A} \in W A$ and $\mathfrak{A}$ is complete and atomic, then $\mathfrak{A} \in \mathrm{R} 1$ RRA.

(2) $W A=\mathrm{SR} 1 R R A$.

Proof. (1) By 5.13, $\mathfrak{A} \cong \mathfrak{R} \mathfrak{l}_{a} \mathfrak{B}$ for some complete atomic $\mathfrak{B} \in W A$ satisfying 5.13(*), but $\mathfrak{B} \in R R A$ by 5.19 and $1.9(2)$, so $\mathscr{A} \in I \mathrm{Rl} R R A=\mathrm{Rl} I R R A=\mathrm{Rl} R R A$ by $5.7(2)$.

(2) Every $W A$ is a subalgebra of a complete atomic $W A$ by 4.2 , so $W A \subseteq \mathrm{SR} 1$ $R R A$ by part (1). On the other hand we have $R R A \subseteq W A$ by $1.9(1), 1.14$, and 1.3 , so $\mathrm{SR} 1 R R A \subseteq \mathrm{SR} 1 W A=\mathrm{S} W A=W A$ by 5.7(6), 5.8, and 1.5 .

D. Resek proved an analogue of 5.20 for cylindric algebras in [15]. Henkin and Resek show in [5] that there are atomic subalgebras of relativized cylindric set algebras which are not relativized cylindric algebras. A study of their example and the connections between $W A$ 's, $S A$ 's, and cylindric algebras makes it seem possible that there are atomic $W A$ 's which are not relativized $S A$ 's.

Problem 5.21. Characterize R1 RRA. Is it an elementary class? Is every atomic $W A$ in $\mathrm{R} 1 S A$ ? Is Rl $S A=\mathrm{R} 1 R R A$ ?

\section{BIBLIOGRAPHY}

1. L. H. Chin and A. Tarski, Distributive and modular laws in the arithmetic of relation algebras, Univ. Calif. Publ. Math. (N.S.) 1 (1951), 341-384.

2. G. Grätzer, Universal algebra, 2nd ed., Van Nostrand, Princeton, N. J., 1978.

3. L. Henkin, Internal semantics and algebraic logic. Truth, syntax and modality, Studies in Logic, vol. 68 (H. Leblanc, ed.), North-Holland, Amsterdam, 1973, pp. 111-127.

4. L. Henkin, J. D. Monk and A. Tarski, Cylindric algebras. I, North-Holland, Amsterdam, 1971.

5. L. Henkin and D. Resek, Relativization of cylindric algebras, Fund. Math. 82 (1975), 363-383.

6. B. Jónsson and A. Tarski, Boolean algebras with operators. I, Amer. J. Math., 73 (1951), 891-939.

7. _ B Boolean algebras with operators. II, Amer. J. Math. 74 (1952), 127-162.

8. R. C. Lyndon, The representation of relation algebras, Ann. of Math. (2) 51 (1950), 707-729.

9. ___ Relation algebras and projective geometries, Michigan Math. J. 8 (1961), 21-28.

10. R. Maddux, Some nonrepresentable relation algebras, Notices Amer. Math. Soc. 23 (1976), A-431, Abstract A-557.

11. Some sufficient conditions for the representability of relation algebras, Algebra Universalis 8 (1978), 162-172.

12. __ Topics in relation algebras, Doctoral dissertation, University of California, Berkeley, 1978.

13. R. N. W. McKenzie, The representation of relation algebras, Doctoral dissertation, University of Colorado, Boulder, 1966.

14. J. D. Monk, On representable relation algebras, Michigan Math. J. 11 (1964), 207-210.

15. D. Resek, Some results on relativized cylindric algebras, Doctoral dissertation, University of California, Berkeley, 1975.

16. R. Sikorski, Boolean algebras, Springer-Verlag, New York, 1969.

17. A. Tarski, Contributions to the theory of models. III, Nederl. Akad. Wetensch. Proc. Ser. A 58 (1955), $56-64$.

Department of Mathematics, Iowa State University, Ames, Iowa 50011 